\title{
Anti-arthritic activity of aqueous-methanolic extract and various fractions of Berberis orthobotrys Bien ex Aitch
}

\author{
Alamgeer ${ }^{*}$, Ambreen Malik Uttra and Umme Habiba Hasan
}

\begin{abstract}
Background: The roots and stem bark of Berberis orthobotrys (Berberidaceae) have long been used traditionally to treat joint pain. Though, it has not been pharmacologically assessed for rheumatoid arthritis. The current study explores anti-arthritic activity and phytochemical analysis of aqueous-methanolic extract (30:70) and fractions (ethyl acetate, $n$-butanol, and aqueous) of Berberis orthobotrys roots.

Methods: Anti-arthritic potential was evaluated in vitro using protein denaturation (bovine serum albumin and egg albumin) and membrane stabilization methods at $12.5-800 \mu \mathrm{g} / \mathrm{ml}$ concentration and in vivo via turpentine oil, formaldehyde and Complete Freund Adjuvant (CFA) models at 50, 100 and $150 \mathrm{mg} / \mathrm{kg}$ doses. Also, in vitro antioxidant ability was appraised by reducing power assay. Moreover, total flavonoid content, Fourier transform infrared spectroscopy and High performance liquid chromatography of $n$-butanol fraction were performed.

Results: The results revealed concentration dependent inhibition of albumin denaturation and notable RBC membrane stabilization, with maximum results obtained at $800 \mu \mathrm{g} / \mathrm{ml}$. Similarly, plant exhibited dose dependent anti-arthritic effect in turpentine oil and formaldehyde models, with maximum activity observed at $150 \mathrm{mg} / \mathrm{kg}$. The results of CFA model depicted better protection against arthritic lesions and body weight alterations. Also, B.orthobotrys remarkably ameliorated altered hematological parameters, rheumatoid factor and positively modified radiographic and histopathological changes. Additionally, plant exhibited remarkable anti-oxidant activity. Moreover, phytochemical analysis revealed polyphenols and flavonoids.
\end{abstract}

Conclusion: Taken together, these results support traditional use of B.orthobotrys as potent anti-arthritic agent that may be proposed for rheumatoid arthritis treatment.

Keywords: Arthritis, Anti-oxidant, Berberidaceae, Phytochemical

\section{Background}

Rheumatoid arthritis (RA) is an advancing, lingering, and a crippling disorder characterized by swelling, pain, and synovial joints stiffness. The exact etiology of this devastating disease is unknown. However, it is strongly linked to autoimmune reaction triggered by various genetic and external factors [1]. The normal use of available prevailing therapies constantly induce harmful consequences, which with the time may neutralize valuable outcomes. Plant drugs are favored over conventional medicines by patients

\footnotetext{
* Correspondence: alam_yuchi@yahoo.com

Laboratory of Cardiovascular Research and Integrative Pharmacology,

Department of Pharmacology, Faculty of Pharmacy, University Of Sargodha,

Sargodha, Pakistan
}

because of unremitting quality of malady, fear of surgery, terrible morbidness, ever-growing medicinal cost, trivial reaction to established drugs and disadvantages of novel drugs. These herbal remedies diminish the manifestations of illness and raise the worth of life [2].

The genus Berberis (Berberidaceae) called "Zereshk" in Persian language comprises around 500 species. A few Berberis species i.e., B. crataegina, B. aristata, B. vul $B$ calliobotrys have been described to possess shrub. It has been found in various parts of world. In Pakistan, it is widely distributed in Northern areas including Gilgit-Baltistan, where residents usually use 
roots and stem bark in powder/pills form for treating joint pain [8].

Preceding phytochemical studies have reported aporphine-benzylisoquinoline alkaloids namely berberine, berbamine, oxyacanthine, pakistanine, pakistanamine, kalashine and chitraline in B. orthobotrys [9]. Among these, berberine has been stated to have potential therapeutic implication in the treatment of RA due to its anti-proliferative effect against rheumatoid arthritis fibroblast like synoviocytes (RAFLS) [10]. Also, berberine has been reported to inhibit chronic inflammatory responses $[6,11-13]$. In addition, berbamine exhibits immunosuppressive effect [14].

Hence, on account of traditional use of $B$. orthobotrys for joint pain, and the above mentioned effects of berberine and berbamine, the main constituents of $B$. orthobotrys against chronic inflammatory and immune responses, it was thought worthwhile to scientifically evaluate anti-arthritic activity of $B$. orthobotrys by means of both in vitro and vivo procedures, as no scientific data regarding anti-arthritic effect of this plant is existing to our utmost information.

\section{Methods}

\section{Plant material}

The roots of Berberis orthobotrys (Local name: Ishkeen) were collected from district Gilgit, Pakistan during the month of June, 2011 by Dr. Alamgeer, resident of village Shikiyote, District Gilgit. It was identified and authenticated by Dr. Shair Wali Khan, Assistant Professor Botany, Karakorum International University Gilgit Baltistan Pakistan. A voucher no. (BO-15-12) had been deposited in herbarium, Faculty of Pharmacy, University of Sargodha for future reference.

\section{Preparation of extract}

The cold maceration process was used to prepare aqueous-methanolic (30:70) extract of B.orthobotrys root. The grounded plant material $(2 \mathrm{~kg})$ was soaked in $5 \mathrm{~L}$ of water-methanol mixture (30:70) for $72 \mathrm{~h}$ at room temperature with occasional stirring on daily basis. After three days, whole material was filtered through Whattman-1 filter paper and filtrate evaporated under reduced pressure on rotary evaporator. The crude extract was then air-dried to attain a solid mass, giving a yield of $18 \%$. Afterwards, $100 \mathrm{~g}$ of plant extract was mixed with $500 \mathrm{ml}$ of distilled water and partitioned with equal volume $(500 \mathrm{ml})$ of ethyl acetate for three consecutive times. This provided $13 \mathrm{~g}$ of ethyl acetate fraction of $B$. orthobotrys, after collecting and evaporating ethyl acetate layer. The residual aqueous layer was further extracted with $500 \mathrm{ml}$ of $n$-butanol for three consecutive times, thus providing $26.1 \mathrm{~g}$ of $B$. orthobotrys $n$-butanol fraction, after collecting and evaporating butanol layer. The remaining aqueous layer was evaporated and resulted in $60.9 \mathrm{~g}$ of aqueous fraction of $B$. orthobotrys [15]. The aqueous-methanolic plant extract and fractions were dissolved in distilled water for use in in vitro and vivo experiments.

\section{Chemicals}

Bovine Serum Albumin (Sigma-Aldrich, USA), Fresh hen's egg albumin, Complete Freund's adjuvant (Sigma-Aldrich, USA), Formaldehyde (VWR, International Ltd), Turpentine oil (UNI-CHEM), Aspirin (UNI-CHEM), Diclofenac Sodium (Sigma-Aldrich, USA), Ascorbic acid (MERCK, Darmstadt, Germany). All the other chemicals used were of analytical grade.

\section{Animals}

Sprague Dawley rats (either sex), weighing 200$300 \mathrm{~g}$ were purchased from University of Agriculture, Faisalabad, Pakistan. They were housed in stainless steel cages under controlled room temperature $\left(25 \pm 2{ }^{\circ} \mathrm{C}\right)$, with $12 \mathrm{~h}$ light/dark cycle and allowed ad libitium access to diet and water and received human care according to requirements of National Institute of Health (NIH) guidelines for care and use of laboratory animals. All the study protocols were approved by Institutional Animal Ethics Committee, Faculty of Pharmacy, University of Sargodha (Approval No. 20A25 IEC UOS). All the experiments performed complied with the rules of National Research Council [16].

\section{Pharmacological investigations}

Evaluation of anti-arthritic effect of Berberis orthobotrys on inhibition of protein denaturation using bovine serum albumin (BSA)

The reaction mixture $(0.5 \mathrm{ml})$ contained $0.45 \mathrm{ml} \mathrm{BSA}$ (5\% aqueous solution) and $0.05 \mathrm{ml}$ of different concentrations $(12.5,25,50,100,200,400,800 \mu \mathrm{g} / \mathrm{ml})$ of B.orthobotrys crude extract, fractions and aspirin (reference drug), correspondingly. Each solution was attuned to $\mathrm{pH} 6.3$ by $1 \mathrm{~N} \mathrm{HCl}$. The samples were incubated at $37{ }^{\circ} \mathrm{C}$ for $20 \mathrm{~min}$ and heated at $57^{\circ} \mathrm{C}$ for $30 \mathrm{~min}$. Then phosphate buffer $(2.5 \mathrm{ml})$ was added and absorbance was measured at $660 \mathrm{~nm}$ via spectrophotometer. For test control $0.05 \mathrm{ml}$ distilled water was used instead of B.orthobotrys while product control lacked BSA [7]. The percentage inhibition of protein denaturation was deliberated by following formula:

Percentage inhibition

$$
=100-\left[\frac{\text { Abs Test Solution-Abs Product Control }}{\text { Abs Test Control }}\right] \times 100
$$

Abs $=$ Absorbance 
Evaluation of anti-arthritic effect of Berberis orthobotrys on inhibition of protein denaturation using egg albumin

The reaction mixture $(5 \mathrm{ml})$ included egg albumin $(0.2 \mathrm{ml})$, phosphate buffered saline, $2.8 \mathrm{ml}(\mathrm{pH} \mathrm{6.4)}$ ) and $2 \mathrm{ml}$ of B.orthobotrys (crude extract and fractions respectively) and diclofenac sodium at various concentrations $(12.5,25,50,100,200,400$ and $800 \mu \mathrm{g} / \mathrm{ml})$, respectively. Equal volume of double-distilled water served as control. The mixtures were incubated at $37 \pm 2{ }^{\circ} \mathrm{C}$ in a Biochemical oxygen demand (BOD) incubator for $15 \mathrm{~min}$ and then heated at $70{ }^{\circ} \mathrm{C}$ for $5 \mathrm{~min}$. Their absorbance was measured at $660 \mathrm{~nm}$ [7]. The percentage inhibition of protein denaturation was appraised using undermentioned formula:

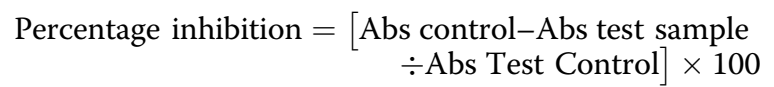

Abs $=$ Absorbance.

\section{Evaluation of anti-arthritic effect of Berberis orthobotrys on human red blood cell (HRBC) membrane stabilization}

The underlying method was used to perform membrane stabilizing activity [7]. Blood was taken from healthy human volunteers (informed consent was taken from blood donors to participate in the study, which was approved by Institutional Human Ethics Committee, Faculty of Pharmacy, University of Sargodha (Approval No. 12H09 IHEC UOS)), mixed with equivalent volume of sterilized Alsevers solution and centrifuged at $3000 \mathrm{rpm}$. The packed cells were washed with isosaline and $10 \% \mathrm{v} / \mathrm{v}$ suspension of red blood cells was prepared and used for study. Test solution consisted of $1 \mathrm{ml}$ of phosphate buffer ( $\mathrm{pH} 7.4,0.15 \mathrm{M})$, hypotonic saline $(2 \mathrm{ml}), 0.5 \mathrm{ml}$ of B.orthobotrys crude extract, fractions and diclofenac sodium at various concentrations $(12.5,25,50,100,200$, 400 and $800 \mu \mathrm{g} / \mathrm{ml}$ ), respectively and 10\% HRBC $(0.5 \mathrm{ml})$. Test control solution comprised phosphate buffer $(1 \mathrm{ml})$, distilled water $(2 \mathrm{ml})$ and $10 \% \mathrm{HRBC}$ $(0.5 \mathrm{~mL})$ in isotonic saline. Assay mixtures were incubated at $37{ }^{\circ} \mathrm{C}$ for $30 \mathrm{~min}$, centrifuged at $3000 \mathrm{rpm}$, supernatant was decanted and hemoglobin content was estimated at $560 \mathrm{~nm}$ spectrophotometrically. The percentage membrane stabilization was projected using following formula:

$$
\begin{aligned}
\text { Percentage protection } & =100-[(\text { Absorbance sample } \\
& \div \text { Absorbance control }) \times 100]
\end{aligned}
$$

Evaluation of anti-arthritic activity of B. orthobotrys against turpentine oil induced joint edema in rats

The rats were fasted for $24 \mathrm{~h}$ prior to conducting experiment and were alienated into 14 groups $(n=5)$.
Group I: Arthritic control rats $(3 \mathrm{ml} / \mathrm{kg}$ distilled water). Group II: $100 \mathrm{mg} / \mathrm{kg}$, p.o. aspirin. Group III, IV, V: B.orthobotrys crude extract at 50, 100, $150 \mathrm{mg} /$ kg, p.o. respectively. Group VI, VII, VIII: $n$-butanol fraction (50, 100, $150 \mathrm{mg} / \mathrm{kg}$, p.o. correspondingly). Group IX, X, XI: Ethyl acetate fraction at 50, 100, $150 \mathrm{mg} / \mathrm{kg}$, p.o. accordingly. Group XII, XIII, XIV: Aqueous fraction $(50,100,150 \mathrm{mg} / \mathrm{kg}$, p.o. in that order). Inflammatory joint edema was developed by injecting $0.02 \mathrm{ml}$ of turpentine oil into synovial cavity of right knee joint, $30 \mathrm{~min}$ following drug administration. Joint diameter was recorded with digital vernier calliper at hourly intervals for $6 \mathrm{~h}$ [7]. Percentage inhibition of knee joint edema by plant extract and aspirin was computed by correlating with untreated arthritic control rats, using following formula.

$$
\text { Percentage inhibition }=\frac{\mathrm{VC}-\mathrm{VT}}{\mathrm{VC}} \times 100
$$

$\mathrm{VC}=$ Joint edema of control group; $\mathrm{VT}=$ Joint edema of the test group.

\section{Evaluation of anti-arthritic activity of B. orthobotrys against formaldehyde induced arthritis in rats}

The rats were distributed into 14 groups $(n=5)$. Group I: Arthritic control rats $(3 \mathrm{ml} / \mathrm{kg}$ distilled water). Group II: $100 \mathrm{mg} / \mathrm{kg}$ aspirin. Group III, IV, $\mathrm{V}$ : B.orthobotrys crude extract $(50,100,150 \mathrm{mg} / \mathrm{kg}$, p.o. respectively). Group VI, VII, VIII: n-butanol fraction $(50,100,150 \mathrm{mg} / \mathrm{kg}, \quad$ p.o. respectively). Group IX, X, XI: Ethyl acetate fraction (50, 100, $150 \mathrm{mg} / \mathrm{kg}$, p.o. correspondingly). Group XII, XIII, XIV: Aqueous fraction $(50,100,150 \mathrm{mg} / \mathrm{kg}$, p.o. in that order). On day 1, $30 \mathrm{~min}$ subsequent to drug administration, arthritis was induced by sub plantar injection of $2 \%$ formaldehyde solution $(0.1 \mathrm{ml})$ and recurrent induction on day 3. Drug treatment was sustained for 10 days. Arthritis was evaluated by checking mean increase in paw diameter for 10 days via digital vernier calliper [7]. Percentage inhibition of paw edema was worked out as described previously.

Evaluation of anti-arthritic activity of $B$. orthobotrys against complete Freund adjuvant (CFA) induced arthritis in rats

The rats were allocated into 7 groups $(n=5)$. Group I: Normal control rats $(3 \mathrm{ml} / \mathrm{kg}$ distilled water $)$. Group II: Arthritic control rats $(3 \mathrm{ml} / \mathrm{kg}$ distilled water). Group III: Aspirin (100 mg/kg, p.o.). Group IV: B. orthobotrys crude extract $(150 \mathrm{mg} / \mathrm{kg}$, p.o.). Group V: $n$-butanol fraction (150 mg/kg, p.o.). Group VI: Ethyl acetate fraction (150 mg/kg, p.o.). Group 
VII: Aqueous fraction (150 mg/kg, p.o.). Arthritis was induced by injecting $0.05 \mathrm{ml}$ of CFA subcutaneously into left foot pad of each rat. The treatments were administered to rats, 1 day ahead of CFA injection and daily treatment continued for 14 days. The injected paw edema was appraised at 1, 5, 10 and 15 days after CFA injection through digital vernier calliper. Percentage inhibition of edema was calculated as described earlier [7].

At 15th day, blood was collected by cardiac puncture for assay of hematologic parameters (RBC, WBC, $\mathrm{Hb}, \mathrm{ESR}$ and Platelet count) and RF level. After that, legs of sacrificed rats were amputated at knee joints and radiographs of control and treated hind paws were taken with computerized radiographic system (Toshiba 630 MA, DS-TA-5A). Then histopathology of ankle joints was performed [7].

\section{Anti-oxidant activity}

\section{Evaluation of reducing power of $B$. orthobotrys}

For the estimation of anti-oxidant activity, B.orthobotrys (extract and fractions, respectively) and standard ascorbic acid at various concentrations $(12.5,25,50$, $100,200,400$ and $800 \mu \mathrm{g} / \mathrm{ml}$ ) were mixed with $2.5 \mathrm{ml}$ phosphate buffer $(0.2 \mathrm{M}, \mathrm{pH} 6.6)$ and $2.5 \mathrm{ml}$ potassium ferricyanide (1\%). Mixture was incubated at $50{ }^{\circ} \mathrm{C}$ for $20 \mathrm{~min}$. Then $2.5 \mathrm{ml}$ TCA, trichloroacetic acid (10\%) was added and centrifuged at $3000 \mathrm{rpm}$ for $10 \mathrm{~min} .2 .5 \mathrm{ml}$ of solution was mixed with $2.5 \mathrm{ml}$ distilled water and $0.5 \mathrm{ml}$ ferric chloride solution $(0.1 \%)$. The absorbance was measured at $700 \mathrm{~nm}$. A blank was prepared without adding extract [17]. Reducing power was calculated using below mentioned formula.

\%increase in reducing power

$$
=[\text { A test }-\mathrm{A} \text { blank } / \mathrm{A} \text { blank }] \times 100
$$

A test is the absorbance of test solution; A blank is the absorbance of blank.

\section{Phytochemical investigations of $n$-butanol fraction Total flavonoids content (TFC)}

The total flavonoid content was found out using a previously described method with some modifications [18]. Briefly, $n$-butanol fraction $(0.5 \mathrm{ml})$ was mixed with distilled water $(2.2 \mathrm{ml})$ and $5 \% \mathrm{NaNO}_{2}$ solution $(0.15 \mathrm{ml})$. Then after $6 \mathrm{~min}, 10 \% \mathrm{AlCl}_{3} \cdot 6 \mathrm{H}_{2} \mathrm{O}$ solution $(0.3 \mathrm{ml})$ was added and allowed to stand for $5 \mathrm{~min}$. After that, $1 \mathrm{M} \mathrm{NaOH}(1 \mathrm{ml})$ was added and mixture was mixed by vortexing. Finally, absorbance was measured at $510 \mathrm{~nm}$ through spectrophotometer. Flavonols in $n$-butanol fraction were expressed as quercetin equivalent. Quercetin was used to perform calibration curve (standard solutions of 6.25, 12.5, 25.0, 50.0 and $100.0 \mu \mathrm{g} / \mathrm{ml}$ in $80 \% \mathrm{~V} / \mathrm{V}$ ethanol).

Fourier transformed infrared (FTIR) spectroscopic analysis $1 \mathrm{mg} n$-butanol fraction was taken in a mortar and pestle and grounded with $2.5 \mathrm{mg}$ of dry $\mathrm{KBr}$. The powder was then filled in a $2 \mathrm{~mm}$ internal diameter micro-cup and loaded onto FTIR set at $26 \pm 1{ }^{\circ} \mathrm{C}$. The samples were scanned using Fourier Transform Infrared Spectrometer (Thermo Nicolet Model-6700) in the range of 4000$400 \mathrm{~cm}^{-1}$ [19]. The spectral data obtained were compared with reference chart to identify functional groups present in the sample.

High performance liquid chromatographic (HPLC) analysis The sample preparation was attained by mixing $n$-butanol fraction $(50 \mathrm{mg})$ with distilled water $(16 \mathrm{ml})$ and methanol (24 ml). Thereafter, $6 \mathrm{M} \mathrm{HCl}(10 \mathrm{ml})$ was added. The mixture was kept for $2 \mathrm{~h}$ at $95{ }^{\circ} \mathrm{C}$ in oven, to get aglycons of flavonol glycosides. An HPLC analysis was performed using HPLC model LC-10A (Shimadzu, Japan), fitted with SCL-10A system control unit, Rheodyne injector, two LC10 AT pumps, CTO-10A column oven, SPD-10A UVVisible detector and data acquisition class LC-10 software. A filtered sample $(20 \mu \mathrm{l})$ was injected into HPLC column. Two solvent systems A (H2O: Acetic acid - 94:6, $\mathrm{pH}=2.27$ ) and $\mathrm{B}$ (Acetonitrile 100\%) were used. Isocratic elution of mobile phase was used to perform chromatographic separation (mixture of solvent A and B (50:50 v/ $\mathrm{v})$, which was filtered using $0.45 \mu \mathrm{m}$ membrane under vacuum before use at a flow rate of $1 \mathrm{ml} / \mathrm{min}$ at $30{ }^{\circ} \mathrm{C}$. Detection was performed at a wavelength of $280 \mathrm{~nm}$ [20].

\section{Statistical analysis}

The results were expressed as mean \pm SEM and statistical analysis was carried out by one way ANOVA followed by Dunnett's test and two way ANOVA followed by Bonferroni posttest using Graph Pad Prism 5.0 and $p<0.05$ was considered statistically significant.

\section{Results \\ Effect of B.orthobotrys on inhibition of protein denaturation using BSA}

The inhibitory effects on protein denaturation are shown in Fig. 1. The present findings exhibited a concentration dependent impediment of protein denaturation by B.orthobotrys as well as aspirin throughout the concentration range $(12.5-800 \mu \mathrm{g} / \mathrm{ml})$. Crude extract demonstrated $92.81 \%$ inhibition of protein denaturation at $800 \mu \mathrm{g} / \mathrm{ml}$, which was parallel to aspirin i.e., $97.55 \%$ at $800 \mu \mathrm{g} / \mathrm{ml}$ while, $n$-butanol fraction proved to be most efficacious among all fractions and its results were on a par with plant extract. 


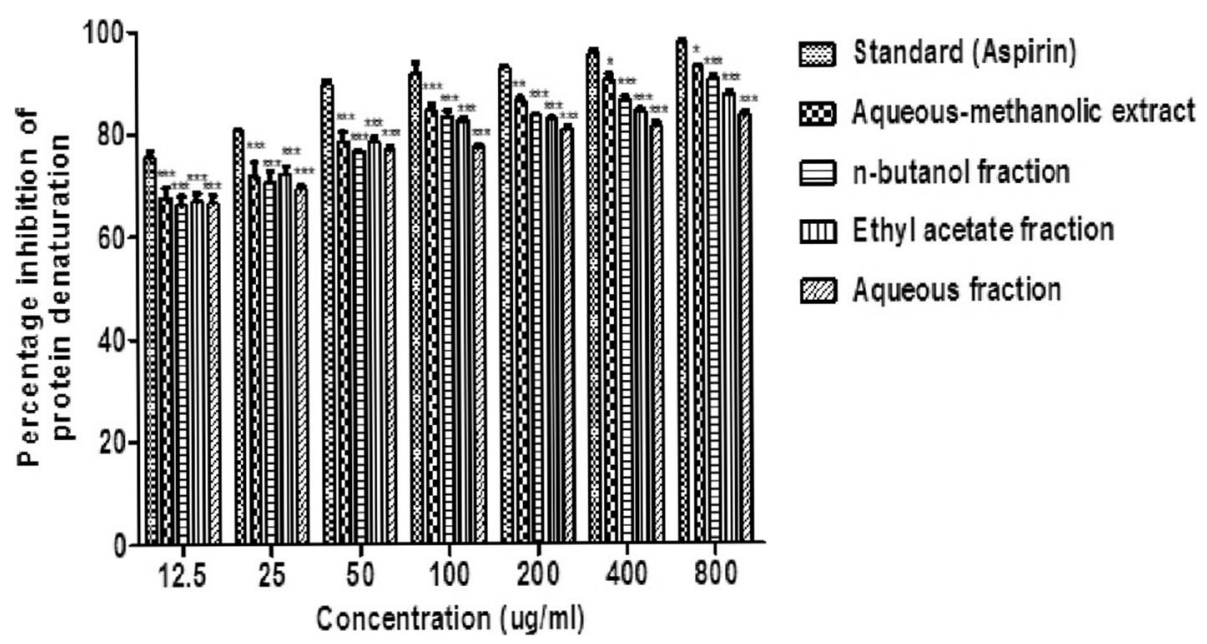

Fig. 1 Effect of B.orthobotrys aqueous-methanolic extract and fractions against protein denaturation using bovine serum albumin. All the values are expressed as mean $\pm \operatorname{SEM}(n=3)$, using two way ANOVA followed by Bonferroni posttest. ${ }^{* *}=p<0.001,{ }^{* *}=p<0.01,{ }^{*}=p<0.05$ vs aspirin

Effect of B.orthobotrys on protein denaturation using fresh hen's egg albumin

B.orthobotrys at several doses $(12.5-800 \mu \mathrm{g} / \mathrm{ml})$ provided considerable protection against denaturation of egg albumin. The results summed up in Fig. 2 point out that crude extract produced $93.30 \%$ inhibition of protein denaturation $(p<0.001)$ at $800 \mu \mathrm{g} / \mathrm{ml}$. Among the fractions, $n$-butanol gave maximum results and showed 92.60\% $(p<0.001)$ protection at $800 \mu \mathrm{g} / \mathrm{ml}$ whereas, diclofenac sodium brought about $99.19 \%$ suppression of protein denaturation at $800 \mu \mathrm{g} / \mathrm{ml}$.

\section{Effect of B.orthobotrys on membrane stabilization}

In membrane stabilization model, there was a dose dependent increment in percentage protection $(p<0.001)$ for all concentrations $(12.5-800 \mu \mathrm{g} / \mathrm{ml})$ of crude extract and fractions albeit, it was much less than that of diclofenac sodium. The protective outcome detected with crude extract was $50.02 \%(p<0.001)$ at $800 \mu \mathrm{g} / \mathrm{ml}$ however, $n$-butanol, most active fraction brought about $48.47 \%$ membrane stabilization at $800 \mu \mathrm{g} / \mathrm{ml}(p<0.001)$. Diclofenac sodium exhibited maximum stabilization of $74.75 \%$ at $800 \mu \mathrm{g} / \mathrm{ml}$ (Fig. 3).

Effect of B.orthobotrys against turpentine oil induced joint edema

The results given in Table 1 indicate that oral treatment with $B$. orthobotrys crude extract and fractions at 50, $100,150 \mathrm{mg} / \mathrm{kg}$ exhibited highly significant $(p<0.001)$ concentration dependent suppression of synovial cavity

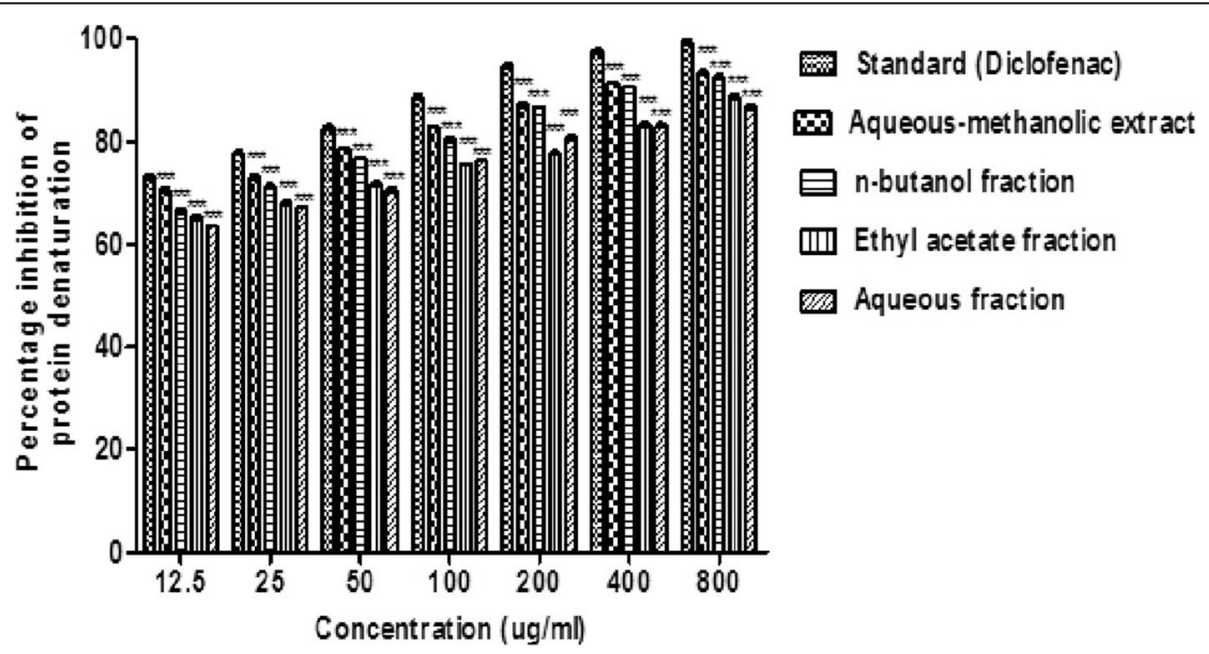

Fig. 2 Effect of B.orthobotrys aqueous-methanolic extract and fractions on protein denaturation using egg albumin. All the values are expressed as mean $\pm \operatorname{SEM}(n=3)$, using two way ANOVA followed by Bonferroni posttest. ${ }^{* *}=p<0.001$ vs diclofenac sodium 


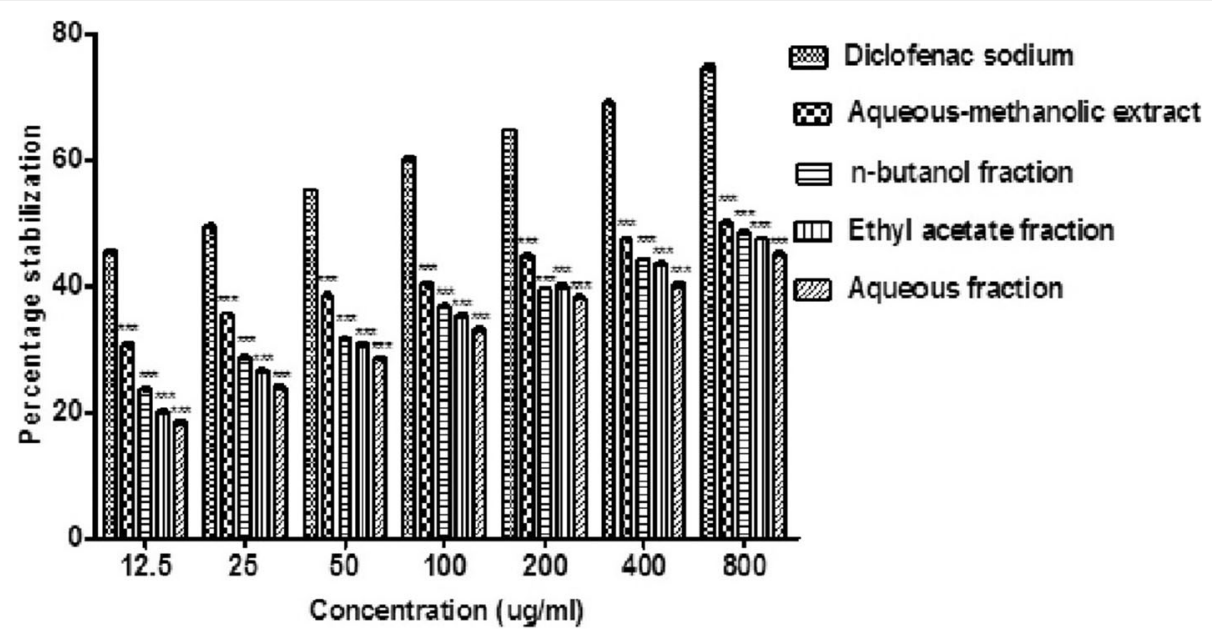

Fig. 3 Effect of $B$. orthobotrys aqueous-methanolic extract and fractions on membrane stabilization. All the values are expressed as mean \pm SEM $(n=3)$, using two way ANOVA followed by Bonferroni posttest. ${ }^{* *}=p<0.001$ vs diclofenac sodium

Table 1 Effect of B.orthobotrys on turpentine oil induced joint edema in rats ( $n=5$, Mean \pm SEM)

\begin{tabular}{|c|c|c|c|c|c|c|}
\hline \multirow[t]{2}{*}{ Treatment groups } & \multicolumn{6}{|c|}{ Increase in joint diameter (mm) } \\
\hline & $1 \mathrm{~h}$ & $2 \mathrm{~h}$ & $3 \mathrm{~h}$ & $4 \mathrm{~h}$ & $5 \mathrm{~h}$ & $6 \mathrm{~h}$ \\
\hline $\begin{array}{l}\text { Arthritic control } \\
(3 \mathrm{ml} / \mathrm{kg})\end{array}$ & $9.44 \pm 0.028$ & $10.59 \pm 0.022$ & $12.34 \pm 0.023$ & $14.98 \pm 0.027$ & $16.38 \pm 0.028$ & $20.96 \pm 0.028$ \\
\hline $\begin{array}{l}\text { Standard Aspirin } \\
(100 \mathrm{mg} / \mathrm{kg})\end{array}$ & $\begin{array}{l}7.02 \pm 0.026^{* * *} \\
(25.63)\end{array}$ & $\begin{array}{l}6.83 \pm 0.026^{* * *} \\
(35.50)\end{array}$ & $\begin{array}{l}6.58 \pm 0.035^{* * *} \\
(46.67)\end{array}$ & $\begin{array}{l}6.37 \pm 0.024^{* * *} \\
(57.34)\end{array}$ & $\begin{array}{l}6.25 \pm 0.028^{* * *} \\
(61.84)\end{array}$ & $\begin{array}{l}6.02 \pm 0.027^{* * * *} \\
(71.27)\end{array}$ \\
\hline $\begin{array}{l}\text { Aqueous-methanolic } \\
\text { extract }(50 \mathrm{mg} / \mathrm{kg})\end{array}$ & $\begin{array}{l}7.40 \pm 0.021^{* * *} \\
(21.61)\end{array}$ & $\begin{array}{l}7.15 \pm 0.021^{* * *} \\
(32.48)\end{array}$ & $\begin{array}{l}6.95 \pm 0.030^{* * *} \\
(43.67)\end{array}$ & $\begin{array}{l}6.87 \pm 0.026^{* * *} \\
(54.13)\end{array}$ & $\begin{array}{l}6.71 \pm 0.026^{* * *} \\
(59.03)\end{array}$ & $\begin{array}{l}6.63 \pm 0.024^{* * * *} \\
(68.36)\end{array}$ \\
\hline $\begin{array}{l}\text { Aqueous-methanolic } \\
\text { extract (100 mg/kg) }\end{array}$ & $\begin{array}{l}7.19 \pm 0.021^{* * *} \\
(23.83)\end{array}$ & $\begin{array}{l}6.87 \pm 0.027^{* * *} \\
(35.12)\end{array}$ & $\begin{array}{l}6.61 \pm 0.022^{* * *} \\
(46.43)\end{array}$ & $\begin{array}{l}6.54 \pm 0.021^{* * *} \\
(56.34)\end{array}$ & $\begin{array}{l}6.33 \pm 0.026^{* * *} \\
(61.35)\end{array}$ & $\begin{array}{l}6.10 \pm 0.024^{* * *} \\
(70.89)\end{array}$ \\
\hline $\begin{array}{l}\text { Aqueous-methanolic } \\
\text { extract ( } 150 \mathrm{mg} / \mathrm{kg})\end{array}$ & $\begin{array}{l}6.84 \pm 0.026^{* * *} \\
(27.54)\end{array}$ & $\begin{array}{l}6.58 \pm 0.025^{* * *} \\
(37.86)\end{array}$ & $\begin{array}{l}6.31 \pm 0.024^{* * *} \\
(48.86)\end{array}$ & $\begin{array}{l}6.14 \pm 0.022^{* * *} \\
(59.01)\end{array}$ & $\begin{array}{l}5.82 \pm 0.023^{* * *} \\
(64.46)\end{array}$ & $\begin{array}{l}5.50 \pm 0.025^{* * *} \\
(73.75)\end{array}$ \\
\hline $\begin{array}{l}\text { n-butanol fraction } \\
(50 \mathrm{mg} / \mathrm{kg})\end{array}$ & $\begin{array}{l}7.75 \pm 0.018^{* * *} \\
(17.90)\end{array}$ & $\begin{array}{l}7.68 \pm 0.019^{* * *} \\
(27.47)\end{array}$ & $\begin{array}{l}8.33 \pm 0.024^{* * *} \\
(32.49)\end{array}$ & $\begin{array}{l}8.29 \pm 0.029^{* * *} \\
(44.65)\end{array}$ & $\begin{array}{l}8.24 \pm 0.029^{* * *} \\
(49.69)\end{array}$ & $\begin{array}{l}8.19 \pm 0.027^{* * *} \\
(60.92)\end{array}$ \\
\hline $\begin{array}{l}\text { n-butanol fraction } \\
(100 \mathrm{mg} / \mathrm{kg})\end{array}$ & $\begin{array}{l}7.51 \pm 0.023^{* * *} \\
(20.44)\end{array}$ & $\begin{array}{l}7.48 \pm 0.023^{* * *} \\
(29.36)\end{array}$ & $\begin{array}{l}7.84 \pm 0.021^{* * *} \\
(36.46)\end{array}$ & $\begin{array}{l}7.76 \pm 0.027^{* * *} \\
(48.19)\end{array}$ & $\begin{array}{l}7.70 \pm 0.025^{* * *} \\
(52.99)\end{array}$ & $\begin{array}{l}7.65 \pm 0.019^{* * *} \\
(63.50)\end{array}$ \\
\hline $\begin{array}{l}\text { n-butanol fraction } \\
(150 \mathrm{mg} / \mathrm{kg})\end{array}$ & $\begin{array}{l}7.32 \pm 0.021^{* * *} \\
(22.45)\end{array}$ & $\begin{array}{l}7.20 \pm 0.021^{* * *} \\
(32.01)\end{array}$ & $\begin{array}{l}7.39 \pm 0.021^{* * *} \\
(40.11)\end{array}$ & $\begin{array}{l}7.21 \pm 0.021^{* * *} \\
(51.86)\end{array}$ & $\begin{array}{l}7.04 \pm 0.028^{* * *} \\
(57.02)\end{array}$ & $\begin{array}{l}6.90 \pm 0.023^{* * *} \\
(67.08)\end{array}$ \\
\hline $\begin{array}{l}\text { Ethyl acetate fraction } \\
(50 \mathrm{mg} / \mathrm{kg})\end{array}$ & $\begin{array}{l}7.96 \pm 0.029 * * * \\
(15.67)\end{array}$ & $\begin{array}{l}7.86 \pm 0.028^{* * *} \\
(25.77)\end{array}$ & $\begin{array}{l}8.44 \pm 0.028^{* * *} \\
(31.60)\end{array}$ & $\begin{array}{l}8.41 \pm 0.031^{* * *} \\
(43.85)\end{array}$ & $\begin{array}{l}8.05 \pm 0.020^{* * *} \\
(50.85)\end{array}$ & $\begin{array}{l}9.10 \pm 0.023^{* * *} \\
(56.58)\end{array}$ \\
\hline $\begin{array}{l}\text { Ethyl acetate fraction } \\
(100 \mathrm{mg} / \mathrm{kg})\end{array}$ & $\begin{array}{l}7.74 \pm 0.021^{* * *} \\
(18.00)\end{array}$ & $\begin{array}{l}7.71 \pm 0.026^{* * *} \\
(27.19)\end{array}$ & $\begin{array}{l}7.96 \pm 0.027^{* * *} \\
(35.49)\end{array}$ & $\begin{array}{l}7.80 \pm 0.028^{* * *} \\
(47.93)\end{array}$ & $\begin{array}{l}7.75 \pm 0.025^{* * *} \\
(52.68)\end{array}$ & $\begin{array}{l}8.40 \pm 0.021^{* * *} \\
(59.92)\end{array}$ \\
\hline $\begin{array}{l}\text { Ethyl acetate fraction } \\
(150 \mathrm{mg} / \mathrm{kg})\end{array}$ & $\begin{array}{l}7.43 \pm 0.027^{* * *} \\
(21.29)\end{array}$ & $\begin{array}{l}7.40 \pm 0.026^{* * *} \\
(30.12)\end{array}$ & $\begin{array}{l}7.73 \pm 0.021^{* * *} \\
(37.35)\end{array}$ & $\begin{array}{l}7.48 \pm 0.017^{* * * *} \\
(50.06)\end{array}$ & $\begin{array}{l}7.22 \pm 0.025^{* * *} \\
(55.92)\end{array}$ & $\begin{array}{l}7.70 \pm 0.030^{* * *} \\
(63.26)\end{array}$ \\
\hline $\begin{array}{l}\text { Aqueous fraction } \\
(50 \mathrm{mg} / \mathrm{kg})\end{array}$ & $\begin{array}{l}8.24 \pm 0.023^{* * *} \\
(12.71)\end{array}$ & $\begin{array}{l}8.11 \pm 0.026^{* * *} \\
(23.41)\end{array}$ & $\begin{array}{l}8.60 \pm 0.026^{* * *} \\
(30.30)\end{array}$ & $\begin{array}{l}8.92 \pm 0.030^{* * *} \\
(40.25)\end{array}$ & $\begin{array}{l}9.13 \pm 0.027^{* * *} \\
(44.26)\end{array}$ & $\begin{array}{l}9.58 \pm 0.025^{* * *} \\
(54.29)\end{array}$ \\
\hline $\begin{array}{l}\text { Aqueous fraction } \\
(100 \mathrm{mg} / \mathrm{kg})\end{array}$ & $\begin{array}{l}7.98 \pm 0.024^{* * *} \\
(15.46)\end{array}$ & $\begin{array}{l}7.77 \pm 0.026^{* * *} \\
(26.62)\end{array}$ & $\begin{array}{l}8.10 \pm 0.026^{* * *} \\
(34.35)\end{array}$ & $\begin{array}{l}8.45 \pm 0.027^{* * *} \\
(43.59)\end{array}$ & $\begin{array}{l}7.78 \pm 0.023^{* * *} \\
(52.50)\end{array}$ & $\begin{array}{l}8.99 \pm 0.025^{* * *} \\
(57.10)\end{array}$ \\
\hline $\begin{array}{l}\text { Aqueous fraction } \\
(150 \mathrm{mg} / \mathrm{kg})\end{array}$ & $\begin{array}{l}7.62 \pm 0.021^{* * *} \\
(19.27)\end{array}$ & $\begin{array}{l}7.54 \pm 0.021^{* * *} \\
(28.80)\end{array}$ & $\begin{array}{l}7.69 \pm 0.028^{* * *} \\
(37.68)\end{array}$ & $\begin{array}{l}7.95 \pm 0.023^{* * * *} \\
(46.92)\end{array}$ & $\begin{array}{l}7.23 \pm 0.033^{* * *} \\
(55.86)\end{array}$ & $\begin{array}{l}8.30 \pm 0.027^{* * * *} \\
(60.40)\end{array}$ \\
\hline
\end{tabular}

Values in the parenthesis represent percentage inhibition of paw edema. The statistical analysis was carried out using two way ANOVA followed by Bonferroni posttest. ${ }^{* *}=p<0.001$ when compared to arthritic control 
Table 2 Effect of B.orthobotrys on formaldehyde induced arthritis in rats ( $n=5$, Mean \pm SEM)

\begin{tabular}{|c|c|c|c|c|c|}
\hline \multirow[t]{2}{*}{ Treatment groups } & \multicolumn{5}{|c|}{ Increase in paw diameter (mm) } \\
\hline & Day 2 & Day 4 & Day 6 & Day 8 & Day 10 \\
\hline $\begin{array}{l}\text { Arthritic control } \\
(3 \mathrm{ml} / \mathrm{kg})\end{array}$ & $8.49 \pm 0.029$ & $10.62 \pm 0.023$ & $12.56 \pm 0.021$ & $14.08 \pm 0.020$ & $16.92 \pm 0.022$ \\
\hline $\begin{array}{l}\text { Standard Aspirin } \\
(100 \mathrm{mg} / \mathrm{kg})\end{array}$ & $\begin{array}{l}4.61 \pm 0.021^{* * *} \\
(45.70)\end{array}$ & $\begin{array}{l}4.95 \pm 0.023^{* * *} \\
(53.38)\end{array}$ & $\begin{array}{l}4.07 \pm 0.019^{* * *} \\
(67.59)\end{array}$ & $\begin{array}{l}3.77 \pm 0.028^{* * *} \\
(73.22)\end{array}$ & $\begin{array}{l}3.63 \pm 0.033^{* * *} \\
(78.54)\end{array}$ \\
\hline $\begin{array}{l}\text { Aqueous-methanolic } \\
\text { extract }(50 \mathrm{mg} / \mathrm{kg})\end{array}$ & $\begin{array}{l}4.76 \pm 0.035^{* * *} \\
(43.93)\end{array}$ & $\begin{array}{l}5.35 \pm 0.026^{* * *} \\
(49.62)\end{array}$ & $\begin{array}{l}4.41 \pm 0.018^{* * *} \\
(64.88)\end{array}$ & $\begin{array}{l}4.12 \pm 0.021^{* * *} \\
(70.73)\end{array}$ & $\begin{array}{l}4.09 \pm 0.019^{* * *} \\
(75.82)\end{array}$ \\
\hline $\begin{array}{l}\text { Aqueous-methanolic } \\
\text { extract (100 mg/kg) }\end{array}$ & $\begin{array}{l}4.51 \pm 0.015^{* * *} \\
(46.87)\end{array}$ & $\begin{array}{l}4.93 \pm 0.023^{* * *} \\
(53.57)\end{array}$ & $\begin{array}{l}4.10 \pm 0.028^{* * *} \\
(67.35)\end{array}$ & $\begin{array}{l}3.72 \pm 0.029^{* * *} \\
(73.57)\end{array}$ & $\begin{array}{l}3.60 \pm 0.027^{* * *} \\
(78.72)\end{array}$ \\
\hline $\begin{array}{l}\text { Aqueous-methanolic } \\
\text { extract ( } 150 \text { mg/kg) }\end{array}$ & $\begin{array}{l}4.28 \pm 0.011^{* * *} \\
(49.58)\end{array}$ & $\begin{array}{l}4.63 \pm 0.016^{* * *} \\
(56.40)\end{array}$ & $\begin{array}{l}3.63 \pm 0.030^{* * *} \\
(71.09)\end{array}$ & $\begin{array}{l}3.28 \pm 0.023^{* * *} \\
(76.70)\end{array}$ & $\begin{array}{l}3.03 \pm 0.017^{* * *} \\
(82.09)\end{array}$ \\
\hline $\begin{array}{l}\text { n-butanol fraction } \\
(50 \mathrm{mg} / \mathrm{kg})\end{array}$ & $\begin{array}{l}4.96 \pm 0.019^{* * *} \\
(41.57)\end{array}$ & $\begin{array}{l}5.46 \pm 0.020^{* * *} \\
(48.58)\end{array}$ & $\begin{array}{l}4.85 \pm 0.025^{* * *} \\
(61.38)\end{array}$ & $\begin{array}{l}4.65 \pm 0.024^{* * *} \\
(66.97)\end{array}$ & $\begin{array}{l}4.54 \pm 0.027^{* * *} \\
(73.16)\end{array}$ \\
\hline $\begin{array}{l}\text { n-butanol fraction } \\
(100 \mathrm{mg} / \mathrm{kg})\end{array}$ & $\begin{array}{l}4.68 \pm 0.023^{* * *} \\
(44.87)\end{array}$ & $\begin{array}{l}5.11 \pm 0.021^{* * *} \\
(51.88)\end{array}$ & $\begin{array}{l}4.41 \pm 0.021^{* * *} \\
(64.88)\end{array}$ & $\begin{array}{l}4.13 \pm 0.021^{* * *} \\
(70.66)\end{array}$ & $\begin{array}{l}4.02 \pm 0.021^{* * *} \\
(76.24)\end{array}$ \\
\hline $\begin{array}{l}\text { n-butanol fraction } \\
(150 \mathrm{mg} / \mathrm{kg})\end{array}$ & $\begin{array}{l}4.38 \pm 0.026^{* * *} \\
(48.40)\end{array}$ & $\begin{array}{l}4.28 \pm 0.020^{* * *} \\
(59.69)\end{array}$ & $\begin{array}{l}3.68 \pm 0.023^{* * *} \\
(70.70)\end{array}$ & $\begin{array}{l}3.55 \pm 0.021^{* * *} \\
(74.78)\end{array}$ & $\begin{array}{l}3.30 \pm 0.023^{* * *} \\
(80.49)\end{array}$ \\
\hline $\begin{array}{l}\text { Ethyl acetate fraction } \\
(50 \mathrm{mg} / \mathrm{kg})\end{array}$ & $\begin{array}{l}5.40 \pm 0.033^{* * *} \\
(36.39)\end{array}$ & $\begin{array}{l}5.70 \pm 0.022^{* * *} \\
(46.32)\end{array}$ & $\begin{array}{l}5.32 \pm 0.030^{* * *} \\
(57.64)\end{array}$ & $\begin{array}{l}5.00 \pm 0.021^{* * *} \\
(64.48)\end{array}$ & $\begin{array}{l}4.91 \pm 0.022^{* * *} \\
(70.98)\end{array}$ \\
\hline $\begin{array}{l}\text { Ethyl acetate fraction } \\
(100 \mathrm{mg} / \mathrm{kg})\end{array}$ & $\begin{array}{l}5.25 \pm 0.021 * * * \\
(38.16)\end{array}$ & $\begin{array}{l}5.50 \pm 0.021^{* * *} \\
(48.21)\end{array}$ & $\begin{array}{l}4.75 \pm 0.017^{* * *} \\
(62.18)\end{array}$ & $\begin{array}{l}4.54 \pm 0.022^{* * *} \\
(67.75)\end{array}$ & $\begin{array}{l}4.48 \pm 0.022^{* * *} \\
(73.52)\end{array}$ \\
\hline $\begin{array}{l}\text { Ethyl acetate fraction } \\
(150 \mathrm{mg} / \mathrm{kg})\end{array}$ & $\begin{array}{l}4.75 \pm 0.020^{* * *} \\
(44.05)\end{array}$ & $\begin{array}{l}5.05 \pm 0.017^{* * *} \\
(52.44)\end{array}$ & $\begin{array}{l}4.37 \pm 0.024^{* * *} \\
(65.20)\end{array}$ & $\begin{array}{l}4.00 \pm 0.021^{* * *} \\
(71.59)\end{array}$ & $\begin{array}{l}3.85 \pm 0.022^{* * *} \\
(77.24)\end{array}$ \\
\hline $\begin{array}{l}\text { Aqueous fraction } \\
(50 \mathrm{mg} / \mathrm{kg})\end{array}$ & $\begin{array}{l}5.88 \pm 0.022^{* * *} \\
(30.74)\end{array}$ & $\begin{array}{l}6.13 \pm 0.021^{* * *} \\
(42.27)\end{array}$ & $\begin{array}{l}5.71 \pm 0.024^{* * *} \\
(54.53)\end{array}$ & $\begin{array}{l}5.37 \pm 0.022^{* * *} \\
(61.86)\end{array}$ & $\begin{array}{l}5.25 \pm 0.024^{* * *} \\
(68.97)\end{array}$ \\
\hline $\begin{array}{l}\text { Aqueous fraction } \\
(100 \mathrm{mg} / \mathrm{kg})\end{array}$ & $\begin{array}{l}5.43 \pm 0.024^{* * *} \\
(36.04)\end{array}$ & $\begin{array}{l}5.96 \pm 0.030^{* * *} \\
(43.87)\end{array}$ & $\begin{array}{l}5.18 \pm 0.027^{* * *} \\
(58.75)\end{array}$ & $\begin{array}{l}4.85 \pm 0.028^{* * *} \\
(65.55)\end{array}$ & $\begin{array}{l}4.75 \pm 0.027^{* * *} \\
(71.92)\end{array}$ \\
\hline $\begin{array}{l}\text { Aqueous fraction } \\
(150 \mathrm{mg} / \mathrm{kg})\end{array}$ & $\begin{array}{l}4.98 \pm 0.023^{* * *} \\
(41.34)\end{array}$ & $\begin{array}{l}5.58 \pm 0.027^{* * *} \\
(47.45)\end{array}$ & $\begin{array}{l}4.47 \pm 0.024^{* * *} \\
(64.41)\end{array}$ & $\begin{array}{l}4.25 \pm 0.021^{* * *} \\
(69.81)\end{array}$ & $\begin{array}{l}4.20 \pm 0.022^{* * *} \\
(75.17)\end{array}$ \\
\hline
\end{tabular}

Values in the parenthesis represent percentage inhibition of paw edema. The statistical analysis was carried out using two way ANOVA followed by Bonferroni posttest. ${ }^{* * *}=P<0.001$ when compared to arthritic control

Table 3 Effect of B.orthobotrys on CFA induced arthritis in rats ( $n=5$, Mean \pm SEM)

\begin{tabular}{|c|c|c|c|c|}
\hline \multirow[t]{2}{*}{ Treatments } & \multicolumn{4}{|c|}{ Increase in paw diameter (mm) } \\
\hline & Day 1 & Day 5 & Day 10 & Day 15 \\
\hline $\begin{array}{l}\text { Arthritic Control } \\
(3 \mathrm{ml} / \mathrm{kg})\end{array}$ & $7.21 \pm 0.035$ & $16.22 \pm 0.029$ & $20.50 \pm 0.024$ & $23.72 \pm 0.023$ \\
\hline Normal Control & $4.19 \pm 0.160^{* * *}$ & $4.24 \pm 0.166^{* * *}$ & $4.24 \pm 0.097^{* * *}$ & $4.27 \pm 0.079^{* * *}$ \\
\hline $\begin{array}{l}\text { Standard Aspirin } \\
(100 \mathrm{mg} / \mathrm{kg})\end{array}$ & $\begin{array}{l}5.90 \pm 0.034^{* * *} \\
(18.16)\end{array}$ & $\begin{array}{l}7.29 \pm 0.030^{* * *} \\
(55.05)\end{array}$ & $\begin{array}{l}6.34 \pm 0.029^{* * *} \\
(69.07)\end{array}$ & $\begin{array}{l}5.83 \pm 0.029^{* * * *} \\
(75.42)\end{array}$ \\
\hline $\begin{array}{l}\text { Aqueous-methanolic } \\
\text { extract ( } 150 \mathrm{mg} / \mathrm{kg})\end{array}$ & $\begin{array}{l}5.13 \pm 0.029^{* * *} \\
(28.84)\end{array}$ & $\begin{array}{l}6.63 \pm 0.029^{* * *} \\
(59.12)\end{array}$ & $\begin{array}{l}5.69 \pm 0.027^{* * *} \\
(72.24)\end{array}$ & $\begin{array}{l}4.51 \pm 0.033^{* * *} \\
(80.98)\end{array}$ \\
\hline $\begin{array}{l}\text { n-butanol fraction } \\
(150 \mathrm{mg} / \mathrm{kg})\end{array}$ & $\begin{array}{l}5.37 \pm 0.030^{* * *} \\
(25.52)\end{array}$ & $\begin{array}{l}6.97 \pm 0.033^{* * *} \\
(57.02)\end{array}$ & $\begin{array}{l}5.75 \pm 0.029^{* * *} \\
(71.95)\end{array}$ & $\begin{array}{l}5.16 \pm 0.032^{* * *} \\
(78.24)\end{array}$ \\
\hline $\begin{array}{l}\text { Ethyl acetate fraction } \\
(150 \mathrm{mg} / \mathrm{kg})\end{array}$ & $\begin{array}{l}5.51 \pm 0.030^{* * *} \\
(23.57)\end{array}$ & $\begin{array}{l}7.68 \pm 0.044^{* * *} \\
(52.65)\end{array}$ & $\begin{array}{l}6.47 \pm 0.032^{* * *} \\
(68.43)\end{array}$ & $\begin{array}{l}5.55 \pm 0.039^{* * *} \\
(76.60)\end{array}$ \\
\hline $\begin{array}{l}\text { Aqueous fraction } \\
(150 \mathrm{mg} / \mathrm{kg})\end{array}$ & $\begin{array}{l}5.82 \pm 0.038^{* * *} \\
(19.27)\end{array}$ & $\begin{array}{l}8.22 \pm 0.034^{* * *} \\
(49.32)\end{array}$ & $\begin{array}{l}6.95 \pm 0.045^{* * *} \\
(66.09)\end{array}$ & $\begin{array}{l}6.02 \pm 0.042^{* * *} \\
(74.62)\end{array}$ \\
\hline
\end{tabular}


swelling when compared to arthritic control. Following 6th $\mathrm{h}$, percentage edema inhibition at $150 \mathrm{mg} / \mathrm{kg}$ plant extract turned out to be $73.75 \%$, which was even more pronounced then that demoed by $100 \mathrm{mg} / \mathrm{kg}$ aspirin i.e., $71.27 \%(p<0.001)$. Likewise, $150 \mathrm{mg} / \mathrm{kg}$ of $n$-butanol, ethyl acetate and aqueous fractions displayed $67.08 \%, 63.26 \%$ and $60.40 \%$ joint stability at 6 th $\mathrm{h}$ respectively.

\section{Effect of B.orthobotrys against formaldehyde induced arthritis}

Results displayed in Table 2 depict that $150 \mathrm{mg} / \mathrm{kg}$ of crude extract and $n$-butanol fraction on 10th day showed more superior repression of paw edema i.e., $82.09 \%$ and
$80.49 \%(p<0.001)$ as compared to $78.54 \%$ reduction in paw edema by $100 \mathrm{mg} / \mathrm{kg}$ aspirin on 10th day. However, ethyl acetate fraction at $150 \mathrm{mg} / \mathrm{kg}$ showed appreciably comparable results to aspirin.

\section{Effect of B.orthobotrys against CFA induced arthritis}

Only the superlative dose $(150 \mathrm{mg} / \mathrm{kg})$ was selected for this method because anti-arthritic efficacy of plant extract has already been justified in aforementioned experiments. Table 3 shows a significant $(p<0.001)$ decrease in paw diameter of treatment groups as compared to CFA control group. Crude extract, $n$-butanol and ethyl acetate fractions at $150 \mathrm{mg} / \mathrm{kg}$ exhibited $80.98 \%, 78.24 \%$ and $76.60 \%$ inhibition of paw edema respectively at the end of

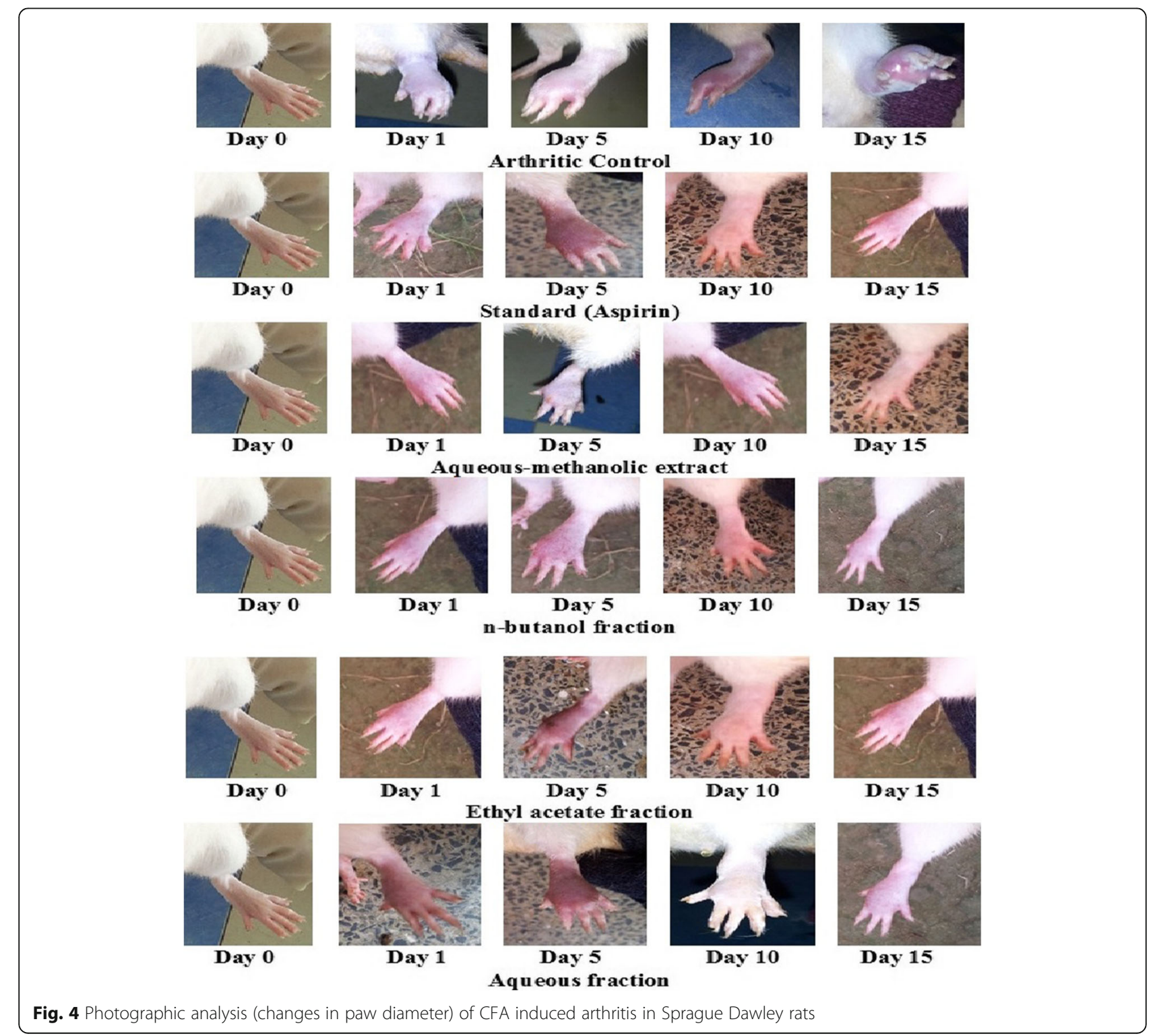


study period. These results were more pronounced than $100 \mathrm{mg} / \mathrm{kg}$ aspirin i.e., $75.42 \%$. Redness and swelling of affected joints were significantly less in treated as compared to arthritic control animals (Fig. 4). A significant $(p<0.001)$ weight gain was observed in treated groups at the end of study (Table 4). The CFA stimulated hematological disturbances and serum RF levels as depicted in Table 5 , were positively altered by $B$.orthobotrys (extract and fractions) and aspirin. Figure 5 shows X-ray radiographs of left hind paws taken on 15 th day. X-rays of normal control group showed no soft tissue swelling, normal bone and cartilage morphology. A limited abnormal pathology was revealed in radiographs of crude extract and $n$-butanol fraction treated rats. Whereas, intense swelling of soft tissues, stern joint destruction, discrete joint space reduction, marked bone resorption and bony erosions seen in arthritic control group were markedly reduced by aspirin and ethyl acetate fraction, however, aqueous fraction explicited mild protective effect. Histopathological changes in joints of arthritic rats are shown in Fig. 6. Histopathology of ankle joint of normal control rat expressed intact morphology of synovium and synovial lining, no inflammation and influx of inflammatory cells. The crude extract and $n$-butanol fraction presented remarkable inhibition of all histological findings of arthritis. The extremely abnormal histology of joint i.e., stern cartilage destruction, disturbed synovial lining, pronounced pannus formation, severe influx of inflammatory cells and erosive changes in cartilage and bone observed in arthritic control group was reversed moderately by aspirin and ethyl acetate fraction and mildly by aqueous fraction.

\section{Reducing power of B.orthobotrys}

The outcomes of anti-oxidant screening described in Fig. 7 depict that B.orthobotrys crude extract displayed $944.59 \%(p<0.001)$ anti-oxidant activity at $800 \mu \mathrm{g} / \mathrm{ml}$. The reductive ability of ascorbic acid was found to be $1636.71 \%$ at $800 \mu \mathrm{g} / \mathrm{ml}$. The most active fraction was found to be $n$-butanol which gave away highly significant $(p<0.001)$ anti-oxidant activity of $855.74 \%$ at $800 \mu \mathrm{g} / \mathrm{ml}$ and its efficacy was closer to crude extract.

\section{Total flavonoid contents in $n$-butanol fraction of B.orthobotrys}

Total flavonoid content in $n$-butanol fraction was found to be $47.45 \mathrm{mg} / \mathrm{g}$ quercetin equivalent.

\section{FTIR analysis of B.orthobotrys $n$-butanol fraction}

Spectroscopic analysis was also performed for $n$-butanol, the most active fraction. A comparison of FTIR spectrum obtained with that of reference chart brought out the existence of phenols, alkanes, carboxylic acids, methyl, carbonyl and carbon fluorine functional groups in $n$-butanol fraction (Table 6 and Fig. 8).

\section{Phenolic profile of B.orthobotrys $\mathrm{n}$-butanol fraction}

The most active fraction of B.orthobotrys, $n$-butanol was selected for phytochemical investigation. The HPLC analysis of $n$-butanol fraction revealed the presence of quercetin, gallic acid, caffeic acid, pcoumaric acid, M-coumaric acid, ferulic acid, trans4-hydroxy-3-methoxy cinnamic acid and sinapic acid (Table 7 and Fig. 9).

Table 4 Effect of B. orthobotrys on body weight of rats in CFA induced arthritis $(n=5$, Mean \pm SEM)

\begin{tabular}{|c|c|c|c|c|}
\hline \multirow[t]{2}{*}{ Treatments } & \multicolumn{4}{|c|}{ Body weight gain (g) } \\
\hline & Day 1 & Day 5 & Day 10 & Day 15 \\
\hline $\begin{array}{l}\text { Arthritic Control } \\
(3 \mathrm{~mL} / \mathrm{kg})\end{array}$ & $262 \pm 3.647$ & $223 \pm 3.906$ & $218 \pm 3.555$ & $208 \pm 4.534$ \\
\hline Normal Control & $269 \pm 6.038^{\mathrm{ns}}$ & $270 \pm 5.544^{* * *}$ & $270 \pm 5.955^{* * *}$ & $274 \pm 6.112^{* * *}$ \\
\hline $\begin{array}{l}\text { Standard Aspirin } \\
(100 \mathrm{mg} / \mathrm{kg})\end{array}$ & $243 \pm 3.040^{* *}$ & $246 \pm 3.597^{* * *}$ & $243 \pm 2.926^{* * *}$ & $245 \pm 3.776^{* * *}$ \\
\hline $\begin{array}{l}\text { Aqueous-methanolic } \\
\text { extract }(150 \mathrm{mg} / \mathrm{kg})\end{array}$ & $265 \pm 3.479^{\text {ns }}$ & $269 \pm 4.176^{* * *}$ & $277 \pm 4.179^{* * *}$ & $285 \pm 4.320^{* * *}$ \\
\hline $\begin{array}{l}\text { n-butanol fraction } \\
(150 \mathrm{mg} / \mathrm{kg})\end{array}$ & $259 \pm 4.841^{\mathrm{ns}}$ & $260 \pm 5.109^{* * *}$ & $261 \pm 4.831^{* * *}$ & $263 \pm 4.802^{* * *}$ \\
\hline $\begin{array}{l}\text { Ethyl acetate fraction } \\
(150 \mathrm{mg} / \mathrm{kg})\end{array}$ & $250 \pm 2.922^{\mathrm{ns}}$ & $251 \pm 2.839^{* * *}$ & $252 \pm 2.379^{* * *}$ & $253 \pm 2.498^{* * *}$ \\
\hline $\begin{array}{l}\text { Aqueous fraction } \\
(150 \mathrm{mg} / \mathrm{kg})\end{array}$ & $242 \pm 2.205^{* *}$ & $242 \pm 2.227^{* *}$ & $243 \pm 2.577^{* * *}$ & $246 \pm 2.782^{* * *}$ \\
\hline
\end{tabular}

The statistical analysis was carried out using two way ANOVA followed by Bonferroni posttest. ${ }^{* *}=p<0.001,{ }^{* *}=p<0.01$, ns $=p>0.05$ compared to arthritic control 


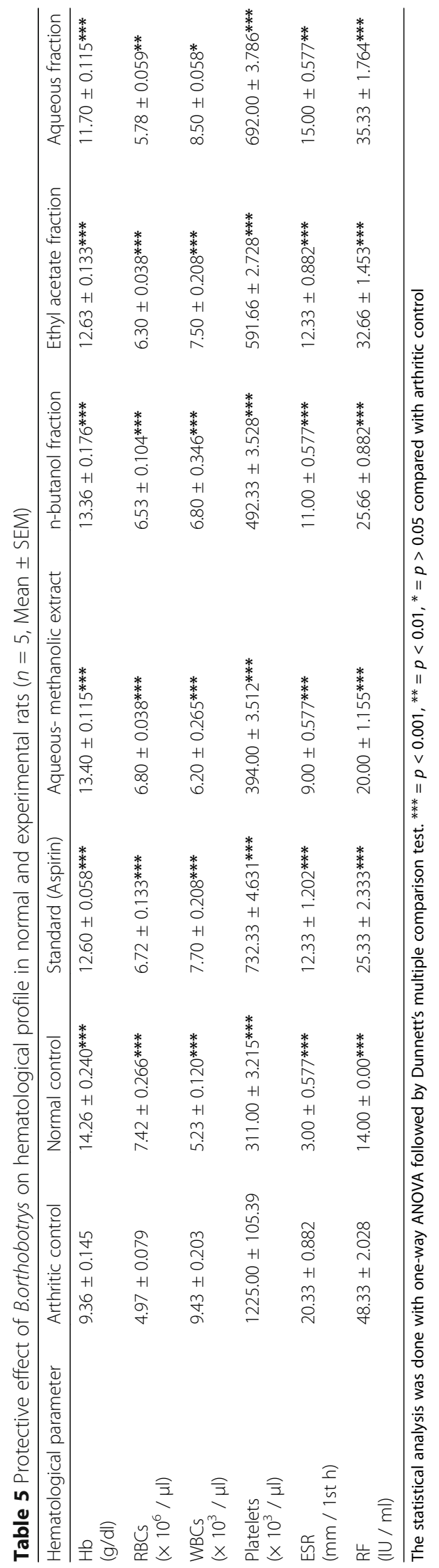




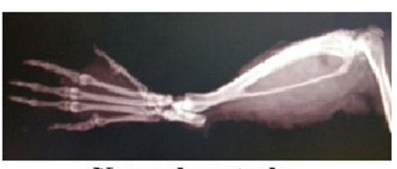

Normal control

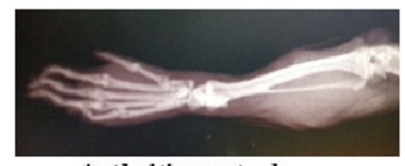

Arthritic control

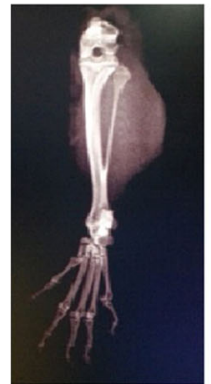

Aspirin

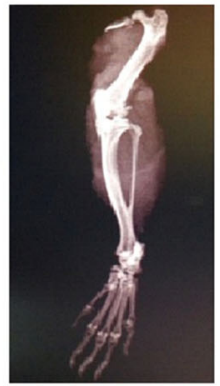

Crude extract

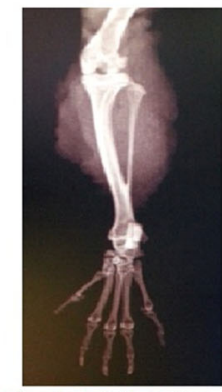

n-butanol fraction Ethyl acetate fraction

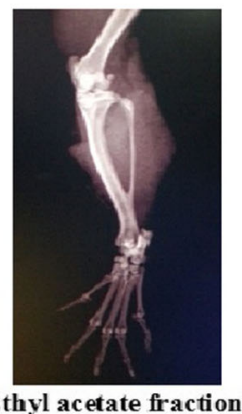

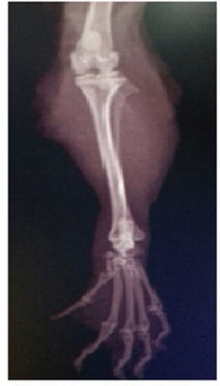

Aqueous fraction

Fig. 5 Radiographic analysis of CFA induced arthritis in Sprague Dawley rats at 15th day of treatment after CFA injection

\section{Discussion}

The present study investigated in vitro and vivo antiarthritic activity and in vitro anti-oxidant potential of aqueous-methanolic extract and fractions (n-butanol, ethyl acetate and aqueous) of B.orthobotrys. Moreover, phytochemical analysis was done using TFC, FTIR and HPLC. The major findings of this study included inhibition of protein (albumin) denaturation, HRBC membrane stabilization, inhibition of paw/joint edema and suppression of certain arthritic parameters by B.orthobotrys. Also, phytochemical studies depicted the presence of flavonoids and phenols.

In protein denaturation, secondary and tertiary structure of proteins is lost by extrinsic stress, heat, organic solvent or strong acid or base [21]. The mechanism of denaturation comprises variation in electrostatic, hydrogen, hydrophobic and disulphide bonding [22]. In the present investigation, plant extract got a higher inhibitory percentage of protein denaturation which was parallel to aspirin also, $n$ butanol fraction exhibited maximum repressing effect as compared to other fractions (Figs. 1 and 2). The increments in absorbance of test samples with respect to control indicated that $B$. orthobotrys has capability to bring down thermal denaturation of protein (albumin).

Since RBC membrane resembles lysosomal membrane so, effect of any agent on RBC stabilization

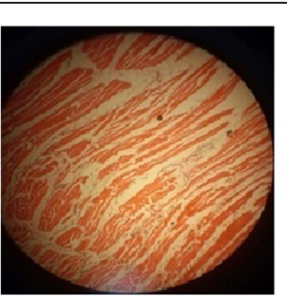

Normal control

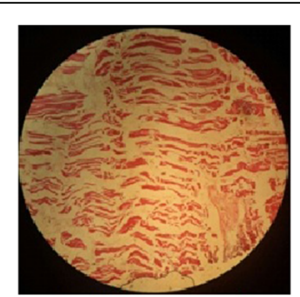

Arthritic control

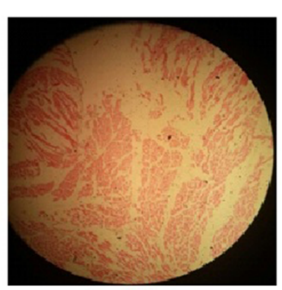

Aspirin

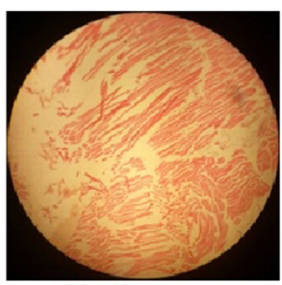

Crude extract

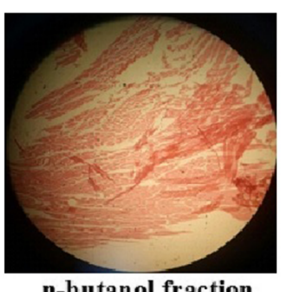

n-butanol fraction

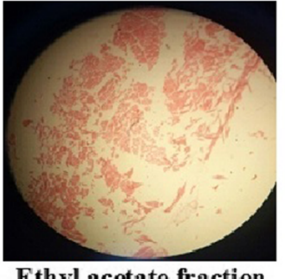

Ethyl acetate fraction

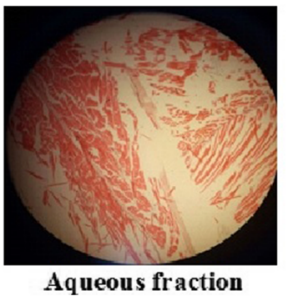

Fig. 6 Histopathological analysis of ankle joint in Sprague Dawley rats at 15th day of treatment after CFA injection 


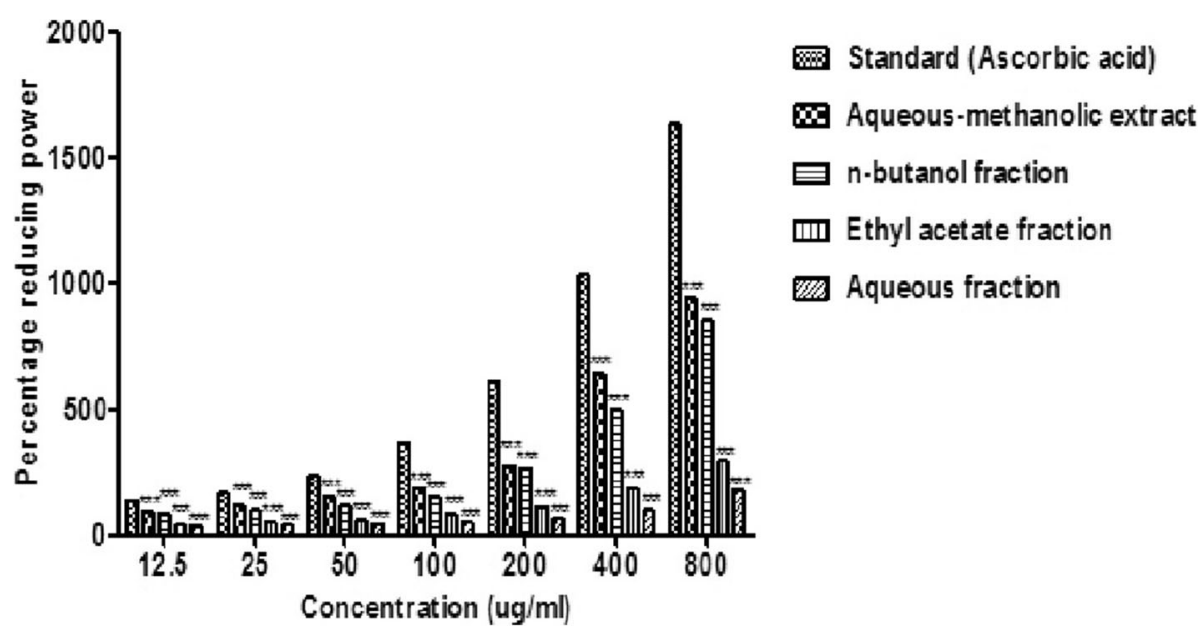

Fig. 7 Effect of B. orthobotrys aqueous-methanolic extract and fractions on reducing power assay. All the values are expressed as mean \pm SEM $(n=3)$, using two way ANOVA followed by Bonferroni posttest. ${ }^{* * *}=(p<0.001)$ vs ascorbic acid

may be projected to lysosomal membrane stabilization [23]. Moreover, strength of RBC's rely on integrity of their membranes and contact of RBC's to hypotonic medium leads to membrane lysis [24]. Also, impairment of lysosome membrane sets off the discharge of lysosomal constituents (bacterial enzymes and proteases) and phospholipase $A_{2}$, inciting phospholipids hydrolysis to produce inflammatory intermediaries [7]. Thus, inhibition of RBC hemolysis in hypotonic media offers an additional mechanism of anti-arthritic effect. In the present study, plant extract and fractions presented an acceptable dose dependent stabilization of RBC membrane (Fig. 3). Membrane stabilizing attribute of B.orthobotrys could be allied with its interfering action on the release of neutrophils lysosomal content. Protective effect on erythrocyte lysis could possibly be acknowledged as an explicit indicator of antiarthritic activity of B.orthobotrys.

Turpentine oil stimulated joint edema has been delineated by enhanced vascular penetrability and

Table 6 Functional groups identified by FTIR spectroscopy in B.orthobotrys $n$-butanol fraction

\begin{tabular}{lll}
\hline Peak & Bond & Functional group \\
\hline $3354.21 \mathrm{~cm}^{-1}-$ & OH Stretching vibrations & Phenols \\
$3334.92 \mathrm{~cm}^{-1}$ & CH Stretching vibrations & Alkanes \\
$2956.87 \mathrm{~cm}^{-1}-$ & $\begin{array}{l}\text { alkanes } \\
2872.01 \mathrm{~cm}^{-1}\end{array}$ & Carboxylic acids \\
$1722.43 \mathrm{~cm}^{-1}$ & $\begin{array}{l}\text { C }=0 \text { carboxylic acid } \\
\text { stretching vibrations }\end{array}$ & Methyl \\
$1459.76 \mathrm{~cm}^{-1}$ & $\mathrm{CH}_{3}$ bending vibrations & Carbonyls \\
$1384.89 \mathrm{~cm}^{-1}-$ & C-O Stretching vibrations & \\
$1367.53 \mathrm{~cm}^{-1}$ & C-F stretching vibrations & Carbon fluorine \\
$1037.7 \mathrm{~cm}^{-1}$ &
\end{tabular}

pronounced vasodilatation in which inflammatory intermediaries are discharged in 3 phases. The initiatory phase is thought to be mediated by serotonin and histamine, intermediate phase by kinin like substances and delayed phase by prostaglandins [25]. Previously aporphine-benzylisoquinoline alkaloids have been reported in B.orthobotrys, namely pakistanine, pakistanamine, kalashine and chitraline, in addition to berberine, berbamine and oxyacanthine [9]. In the present work, plant extract at $150 \mathrm{mg} / \mathrm{kg}$ dose exhibited more pronounced prevention of joint edema as compared to $100 \mathrm{mg} / \mathrm{kg}$ aspirin (Table 1). Impediment of edema intimates potential efficacy of B.orthobotrys on various phases of inflammation, which successively could be on account of presence of alkaloid berberine because formerly, it has also been proclaimed that berberine inhibits vascular permeability [6].

Inhibition of formaldehyde-induced edema is one of the most suitable methods to evaluate anti-proliferative activity and screen anti-arthritic agents. Formaldehyde develops localized inflammation in two phases. In early phase (neurogenic phase), substance $\mathrm{P}$ is discharged, while in late phase (inflammatory phase), histamine, serotonin, bradykinin and prostaglandins are liberated, which ensues in pronounced vasodilation and permeability [40]. It has been proclaimed that drugs that works on CNS inhibit both phases uniformly whilst, peripherally acting drugs inhibit late phase [7]. The results of present study showed that plant extract and $n$-butanol fraction at a dose of $150 \mathrm{mg} / \mathrm{kg}$ subdued proliferative edematous reaction greater than $100 \mathrm{mg} / \mathrm{kg}$ aspirin (Table 2), which tenably could be due to berberine, as B.aristata has previously been reported to inhibit formaldehyde induced arthritis owing to the presence of berberine [5]. 


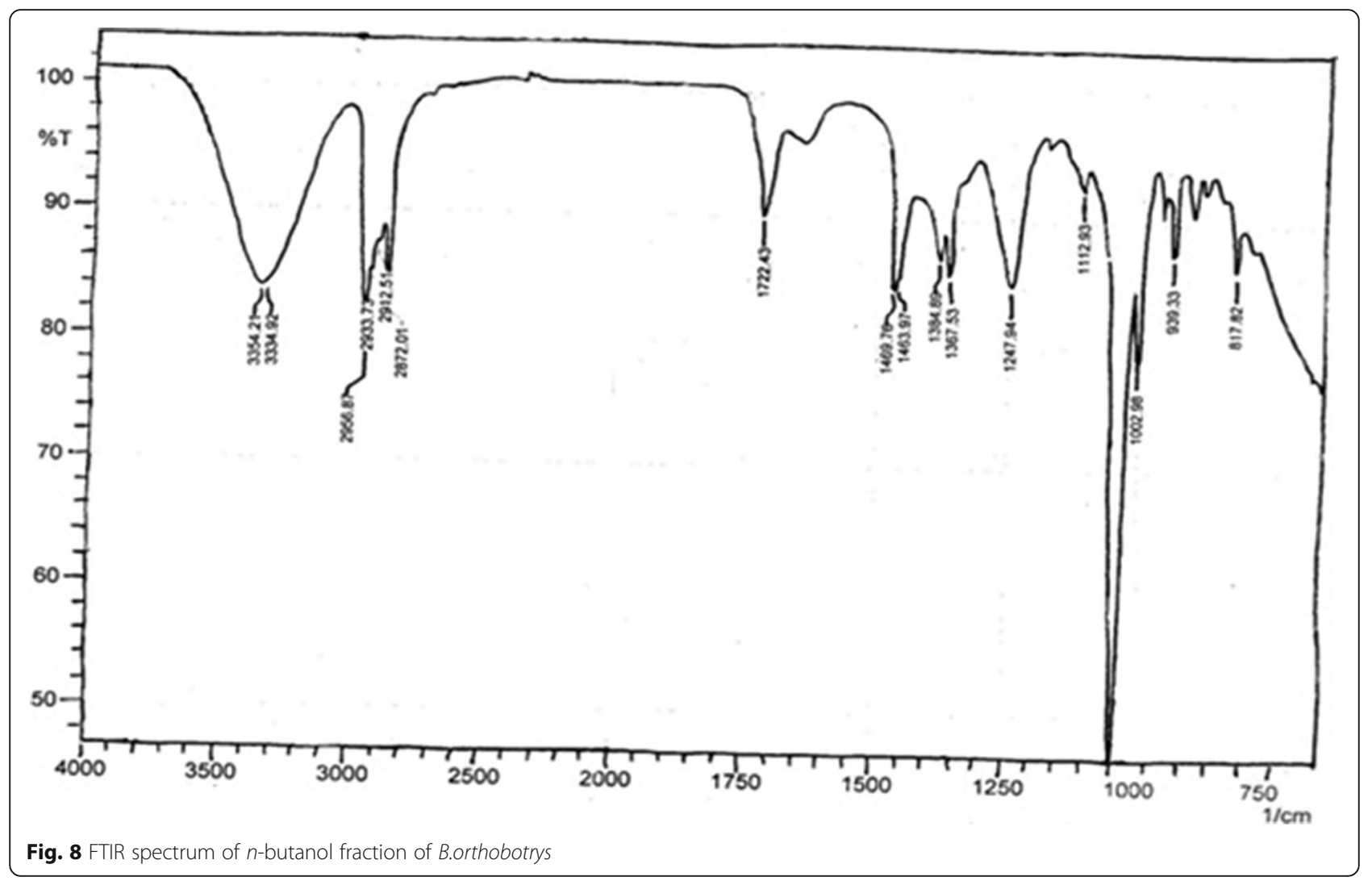

Additionally, impediment of both early and late phases of formaldehyde response show that B.orthobotrys acts on CNS.

In the existing study, primary lesions instigated after 3-5 days of CFA injection while, secondary lesions took place after 11-12 days, featured by inflammation of non-injected hind paw. It has been reported that deformities in $\mathrm{RA}$ initiate due to the production of inflammatory mediators i.e., IFN $\alpha$,

Table 7 Phenolic constituents identified in $n$-butanol fraction of B.orthobotrys

\begin{tabular}{lllll}
\hline Peak No. & RT $(\mathrm{min})$ & Area Count & Amount $(\mathrm{ppm})$ & Compounds \\
\hline 4 & 2.887 & 21.493 & 1.13 & Quercetin \\
6 & 4.927 & 335.728 & 12.08 & Gallic acid \\
12 & 12.767 & 305.143 & 14.03 & Caffeic acid \\
13 & 17.153 & 297.613 & 3.86 & p-coumaric acid \\
14 & 20.760 & 340.918 & 3.56 & M-coumaric acid \\
15 & 21.987 & 152.704 & 10.94 & Ferulic acid \\
17 & 25.280 & 197.301 & 4.53 & $\begin{array}{l}\text { Trans-4-hydroxy- } \\
\text { 3-methoxy cinnamic } \\
\end{array}$ \\
18 & 26.713 & 278.642 & 3.61 & acid \\
\end{tabular}

PDGF and cytokines (IL-1, IL 6 and TNF- $\alpha$ ) [26]. B.orthobotrys significantly inhibited arthritis in chronic stage and protected the joints as clear from histopathology results, which possibly could be due to its impeding action on inflammatory intermediaries as well as the result of immunological protection rendered by plant as supported by previous studies $[13,27]$. RA is also allied with weight loss (rheumatoid cachexia) which may be ascribed to tissue destruction owing to proteolysis of muscle proteins by lysosomal proteases, mediated by $\mathrm{PGE}_{2}$ [7] and decreased absorption of ${ }^{14} \mathrm{C}$-glucose and ${ }^{14} \mathrm{C}$-leucine in rat's intestine [28]. In the present work, evident restoration of rat's body weight with $150 \mathrm{mg} / \mathrm{kg}$ of B.orthobotrys (Table 4) might be credited to reduction in $\mathrm{PGE}_{2}$ levels and increased absorption power of intestine. Additionally, this model gives a chance to analyze hematological alterations. The most frequent extracellular manifestation in RA occurring due to decrease in $\mathrm{Hb}$ and $\mathrm{RBC}$ levels is anemia, which ensues from decreased levels of erythropoietin [29] and reduced plasma iron, induced by IL-1 [7]. Moreover, liberation of IL-1 and TNF $\alpha$ in arthritic state causes rise in WBCs and platelet count [30]. Also, proteins (fibrinogen, $\alpha-$ and $\beta$ - 


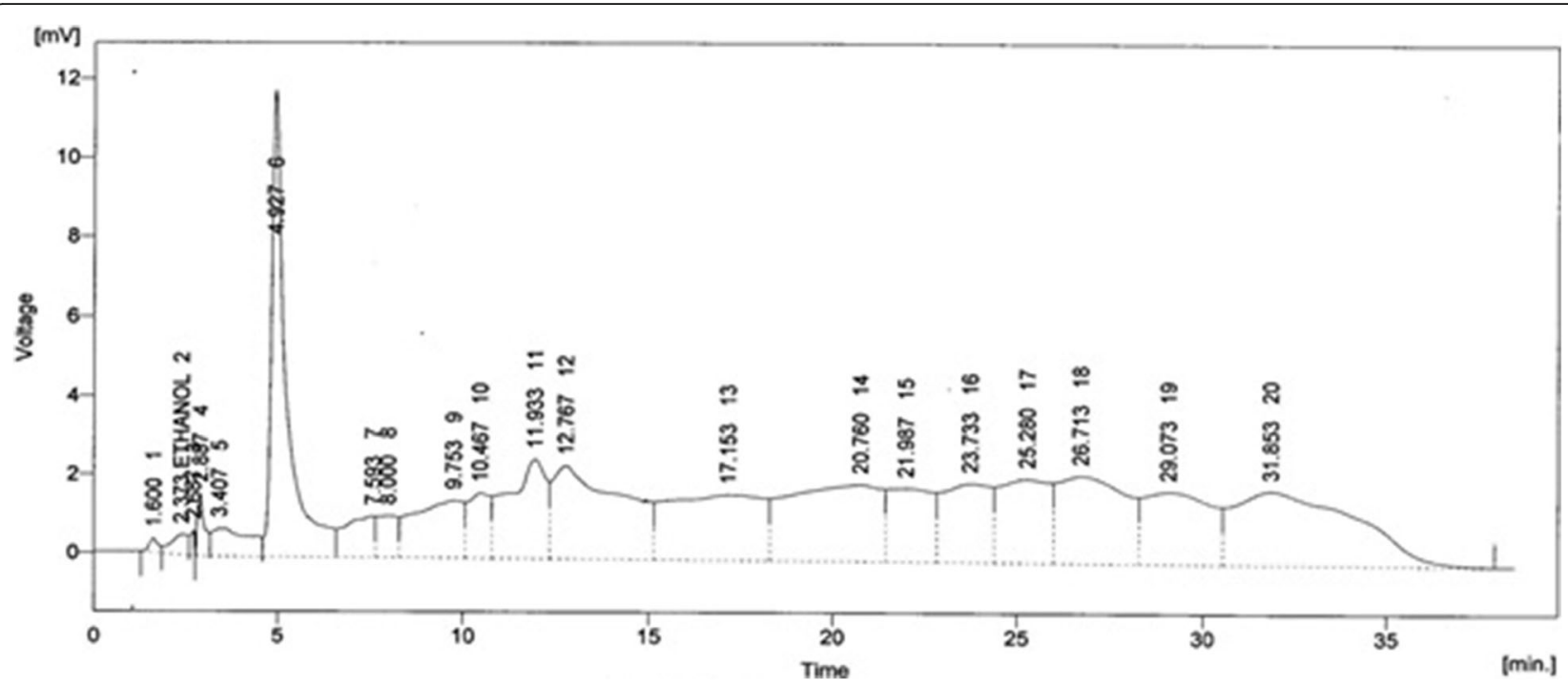

Fig. 9 HPLC chromatogram of $n$-butanol fraction of B.orthobotrys

globulins) formed in reaction to inflammation increases ESR value [31]. Besides this, rheumatoid factor (RF) is a chief serologic marker in arthritis [7], which is an auto-antibody targeted against Fc segment of IgG. The results depicted that B.orthobotrys extract and fractions positively altered hematologic modifications (Table 5). As well, treatment with B.orthobotrys extract and fractions (most prominently $n$-butanol) presented noticeable decrease in radiographic and histological damage and markedly suppressed the modification in joint architecture (Figs. 5 and 6). It can be proposed that inhibition of CFA induced arthritis and its associated alterations might be accredited to the presence of alkaloid berberine and berbamine in B.orthobotrys as berberine has been described to show significant improvement in synovial hyperplasia and inflammatory infiltration via inhibition of TNF- $\alpha$, IL$1 \beta$, IL-6, PGE2, COX-2, NF-k $\beta$, repression of Th17, dendritic cell responses and other signaling pathways [11-13]. Formerly, an analysis carried out on B.vulgaris roots proved a marked repressive effect against CFA induced arthritis owing to the presence of berberine and oxyacanthine [6]. Moreover, berbamine exhibits immunosuppressive effect by stamping down STAT4 appearance and assembling of IFN- $\gamma$ [14].

Reducing ability has been investigated from $\mathrm{Fe}^{+3}$ $\mathrm{Fe}^{+2}$ transformation. Compounds possessing reducing capacity connote that they are electron donors, and have strength to quash the oxidized mediators that are produced as an upshot of lipid peroxidation mechanisms [32]. Extracts of various Berberis spp. (e.g. B. aristata, B. vulgaris, B. croatica, B. microphylla and $B$. lycium) have been described to hold anti-oxidative activities [32-36], which were proved to be associated with phenols and flavonols [34, 36]. Also berberine and berbamine, the main constituents of Berberis spp. have been detected to be antioxidants [37, 38]. Results of present study disclosed that B.orthobotrys owned preeminent puissance to knock off the free radicals (Fig. 7) that can be linked up with the presence of aporphine-benzylisoquinoline alkaloids [9] and reductones (phenols and flavonoids) in B.orthobotrys.

In present investigation as the active fraction was $n$-butanol so, its FTIR analysis revealed the presence of phenols, alkanes, carboxylic acids, methyl, carbonyl and carbon fluorine functional groups (Table 6 and Fig. 8). The presence of phenolics was further confirmed by HPLC and active principles isolated from $n$-butanol fraction were found to be quercetin, gallic acid, caffeic acid, p-coumaric acid, M-coumaric acid, ferulic acid, trans-4-hydroxy-3methoxy cinnamic acid and sinapic acid (Table 7 and Fig. 9). It has formerly been reported that phenolics and flavonoids possess anti-inflammatory and anti-oxidant activities [39]. Previous works have shown that flavonoid quercetin exerts anti-inflammatory, anti-proliferative and anti-oxidative effects [40]. Similarly, gallic acid and phenolic acids i.e., p-coumaric, caffeic, and ferulic acids have been reported as antiinflammatory and free radical scavengers [41, 42].

\section{Conclusion}

In the view of above discussion, it is conceivable that B.orthobotrys Bien ex Aitch has been observed to exert significant anti-arthritic effect in experimental studies. 
Furthermore, $n$-butanol fraction has been avowed to be most potent. Though, exact mechanism of repressing the arthritic state by $B$. orthobotrys is not identified, its beneficial effects on RA could possibly be correlated with the presence of aporphine-benzylisoquinoline alkaloids (berberine, berbamine) detected previously in $B$. orthobotrys and phenols and flavonoids identified in the current study. In summary, this contemporary research lends pharmacological support to reported folkloric usage of B.orthobotrys in the treatment and management of painful arthritic inflammatory conditions. Based on our results, further thorough studies are required for appraisal of exact mechanism of action of B.orthobotrys, determination of pro-inflammatory cytokines level, isolation of active constituents and cellular characterization that could conclusively establish B.orthobotrys as a potentially safer disease modifying agent in the treatment of RA.

\section{Abbreviations}

Abs: Absorbance; BSA: Bovine serum albumin; CFA: Complete freund adjuvant; ESR: Erythrocyte sedimentation rate; FTIR: Fourier transform infrared spectroscopy; Hb: Hemoglobin; HPLC: High performance liquid chromatography; HRBC: Human red blood cell; IFN a: Interferon-a; IL: Interleukin; PDGF: Platelet derived growth factor; PGE2: Prostaglandin E2; RA: Rheumatoid arthritis; RBC: Red blood cell; RF: Rheumatoid factor; TFC: Total flavonoid content; TNF-a: Tumor necrosis factor-a; WBC: White blood cell

\section{Acknowledgements}

Not applicable.

\section{Funding}

The funds for chemicals used in the study were granted by University of Sargodha, Sargodha, Pakistan.

\section{Availability of data and materials}

The datasets used and analyzed during the current study are available from the corresponding author on reasonable request.

\section{Author's contributions}

All the authors contributed equally in research work. All authors read and approved the final manuscript.

\section{Ethics approval and consent to participate}

The experimental procedures were approved by the Institutional Animal Ethics Committee of Faculty of Pharmacy, University of Sargodha (Approval No. $20 \mathrm{~A} 25$ IEC UOS). The consent was taken from human volunteers to donate blood for membrane stabilization assay and Institutional human ethics committee approved the use of human blood for this study (Approval No. 14B IHEC UOS).

\section{Consent for publication}

Not applicable.

\section{Competing interests}

The authors affirm that they have no competing interests.

\section{Publisher's Note}

Springer Nature remains neutral with regard to jurisdictional claims in published maps and institutional affiliations.
Received: 11 April 2017 Accepted: 10 July 2017

Published online: 18 July 2017

\section{References}

1. Lipsky PE, Rheumatoid arthritis. In: Harrison's Principles of Internal Medicine, (Eds. Kasper DL. Fauci AS, Hauser SL, Longo DL \& Jameson JL, McGraw Hill, New York: Braunwald E; 2005. p. 1968

2. Patil KR, Patil CR, Jadhav RB, Mahajan VK, Patil PR, Gaikwad PS. Anti-arthritic activity of Bartogenic acid isolated from fruits of Barringtonia racemosa Roxb. (Lecythidaceae). Evid Based Complement Alternat Med. 2011; (2011): 785245.

3. Yeşilada E, Küpeli E. Berberis crataegina DC. Root exhibits potent antiinflammatory, analgesic and febrifuge effects in mice and rats. J Ethnopharmacol. 2002;79:237-48.

4. Kumar R, Gupta YK, Singh S. Anti-inflammatory and anti-granuloma activity of Berberis aristata DC. In experimental models of inflammation. Indian J Pharmacol. 2016;48:155-61.

5. Biren NS, Nayak BS, Seth AK, Jalalpure SS, Patel KN, Patel MA, Mishra AD. Search for medicinal plants as a source of anti-inflammatory and antiarthritic agents - a review. Pharmacogn Mag. 2006;26:77-86.

6. Ivanovska N, Philipov S. Study on the anti-inflammatory action of Berberis vulgaris root extract, alkaloid fractions and pure alkaloids. Int J Immunopharmacol. 1996;18:553-61.

7. Alamgeer, Hasan UH, Uttra AM, Rasool S. Evaluation of in vitro and in vivo anti-arthritic potential of Berberis calliobotrys. Bangladesh J Pharmacol. 2015: 10: 807-819.

8. Abbas Q, Khan SW, Khatoon S, Hussain SA. Najam ul Hassan S, Hussain a, Qureshi R, Hussain I. Floristic biodiversity and traditional uses of medicinal plants of Haramosh Valley central Karakoram National Park of Gilgit district, Gilgit-Baltistan, Pakistan. J Biodivers Environ Sci. 2014;5:75-86.

9. Hussain SF, Khan L, Sadozai KK, Shamma M. New alkaloids from Berberis orthobotrys. J Nat Prod. 1981;44:274-8.

10. Wang XH, Jiang SM, Sun QW. Effects of berberine on human rheumatoid arthritis fibroblast-like synoviocytes. Exp Biol Med (Maywood). 2011;236: 859-66.

11. Wang Z, Chen Z, Yang S, Wang Y, Huang Z, Gao J, Tu S, Rao Z. Berberine ameliorates collagen induced arthritis in rats associated with antiinflammatory and anti-angiogenic effects. Inflammation. 2014;37:1789.

12. Yang Y, Qi J, Wang Q, Du L, Zhou Y, Yu H, Kijlstra A, Yang P. Berberine suppresses Th17 and dendritic cell responses. Invest Ophthalmol Vis Sci. 2013:54:2516-22.

13. Chatterjee GK, Pal SP. Search for anti-inflammatory agents from Indian medicinal plants: a review. Indian Drugs. 1984;21:413.

14. Ren Y, Lu L, Guo TB, Qiu J, Yang Y, Liu A, Zhang JZ. Novel immunomodulatory properties of berbamine through selective downregulation of STAT4 and action of IFN gamma in experimental autoimmune encephalomyelitis. J Immunol. 2008;181:1491-8.

15. Alamgeer, Chabert P, Akhtar MS, Jabeen Q, Delecolle J, Heintz D, Garo E, Hamburger M, Auger C, Lugnier C, Kim HJ, Oak MH, Schini-Kerth VB. Endothelium-independent vasorelaxant effect of a Berberis orthobotrys root extract via inhibition of phosphodiesterases in the porcine coronary artery. Phytomedicine. 2016; 23: 793-9.

16. National Research Council. Guide for the care and use of laboratory animals. (Washington DC, National Academy Press). 1996

17. Arulpriya $P$, Lalitha $P$, Hemalatha $S$. In vitro antioxidant testing of the extracts of Samanea saman (Jaca.) Merr. Der Chemica Sinica. 2010;1:73-7.

18. Chang LW, Yen WJ, Huang SC, Duh PD. Antioxidant activity of sesame coat. Food Chem. 2002;78:347-54

19. Naumann D, Helm D, Labischinski H, Giesbrecht P. The characterisation of microorganisms by Fourier-transform infrared spectroscopy (FT-IR). In: Modern techniques for rapid microbiological analysis, (Eds. Nelson WH. New York: VCH Publishers; 1991. p. 43.

20. Tokusoglu O, Unal MK, Yildirum Z. HPLC-UV and GC-MS characterization of the flavonols aglycons quercetin, kaempferol and myrecetin in tomato and tomato pastes and other tomato-based products. Acta Chromatogr. 2003;13:196-207.

21. Prasad SB. Yashwant, Aeri V. In vitro anti-inflammatory activity of Raupya (Silver) Bhasma. J Chem Pharm Res. 2013:5:194-7.

22. Grant NH, Alburn HE, Kryzanauskas C. Stabilization of serum albumin by anti-inflammatory drugs. Biochem Pharmacol. 1970;19:715-22. 
23. Gupta SK. Gupta a, Gupta AK, Prakash D, Vedpal. In vitro anti-arthritic activity of ethanolic extract of Callicarpa macrophyla flowers. Int Res J Pharm. 2013:4:160-2.

24. Ferrali M, Signorni C, Ciccoli L, Comporti M. Iron release and membrane damage in erythrocytes exposed to oxidizing agents phenylhydrazine, divicine and isouramil. Biochem J. 1992;285:295-301.

25. Hanson JM, Morley J, Soria-Herrera C. Anti-inflammatory property of 401 (MCD-peptide), a peptide from the venom of bee Apismellifera (L.). $\mathrm{Br} J$ Pharmacol. 1974;50:383-92.

26. Patil MVK, Kandhare AD, Bhise SD. Anti-arthritic and anti-inflammatory activity of Xanthium srtumarium L. ethanolic extract in Freund's complete adjuvant induced arthritis. Biomed Aging Pathol. 2012;2:6-15.

27. Ivanovska N, Philipov S, Hristov M. Influence of berberine on T-cell mediated immunity. Immunopharmacol Immunotoxicol. 1999;21:771-86.

28. Van Arman G. Pathway to adjuvant arthritis. J Fed Proc. 1976:35:2442-6.

29. Hu F, Hepburn HR, Li Y, Chen M, Radloff SE, Daya S. Effect of ethanol and water extracts of Propolis (bee glue) on acute inflammatory animal models. J Ethnopharmacol. 2005;100:276-83.

30. Maria M, Engeniusz M, Miroslaw K, Maria K, Iwona P. Adjuvant induced disease in rats. Clinical findings and morphological and biochemical changes in blood and histological changes in internal organs. Rheumatologia. 1983;21:213-45

31. Van den Hoogen HM, Koes BW, Van Eijk JT, Bouter LM. On the accuracy of history, physical examination, and erythrocyte sedimentation rate in diagnosing low back pain in general practice. Spine. 1995;20:318-27.

32. Mashwani Z, Khan MA, Irum S, Ahmad M. Antioxidant potential of root bark of Berberis lycium Royle. from Galliyat, Western Himalaya, Pakistan. Pak J Bot. 2013; 45: 231.

33. Singh J. Kakkar. Antihyperglycemic and anti-oxidant effect of Berberis aristata root extract and its role in regulating carbohydrate metabolism in diabetic rats. J Ethnopharmacol. 2009;123:22-6.

34. Koncic MZ, Kremer D, Schuly W, Brantner A, Karlovic K, Kalodera Z. Chemical differentiation of Berberis croatica vs B.vulgaris using HPLC fingerprinting. Croat Chem Acta. 2010;83:451-6.

35. Hanachi P. Using HPLC to determination the composition and anti-oxidant activity of Berberis vulgaris. Eur J Sci Res. 2009;29:47-54.

36. Ruiz A, Hermosí-Gutiérrez I, Mardones C, Vergara C, Herlitz E, Vega M, Dorau C, Winterhalter P, Von Baer D. Polyphenols and anti-oxidant activity of Calafate (Berberis microphylla) fruits and other native berries from southern Chile. J Agric Food Chem. 2010;58:6081-9.

37. Guo ZB, Fu JG. Progress of cardiovascular pharmacologic study on berbamine. Zhong Guo Zhong Xi Yi Jie He ZaZhi. 2005;25:765-8.

38. Shirwaikar A, Shirwaikar A, Rajendran K, Punitha ISR. In vitro anti-oxidant studies on the benzyl tetra isoquinoline alkaloid berberine. Biol Pharm Bull. 2006;29:1906-10.

39. Ghasemzadeh A, Ghasemzadeh N. Flavonoids and phenolic acids: role and biochemical activity in plants and human. J Med Plant Res. 2011;5:6697-703.

40. Bischoff SC. Quercetin: potentials in the prevention and therapy of disease. Curr Opin Clin Nutr Metab Care. 2008;11:733-40.

41. Kroes BH, van den Berg AJ, Quarles van Ufford HC, Van Dijk H, Labadie RP, Anti-inflammatory activity of gallic acid. Planta Med 1992; 58: 499-504.

42. Manach C, Scalbert A, Morand C, Remesy C, Jimenez L. Polyphenols: food sources and bioavailability. Am J Clin Nutr. 2004;79:727-47.

\section{Submit your next manuscript to BioMed Central and we will help you at every step:}

- We accept pre-submission inquiries

- Our selector tool helps you to find the most relevant journal

- We provide round the clock customer support

- Convenient online submission

- Thorough peer review

- Inclusion in PubMed and all major indexing services

- Maximum visibility for your research

Submit your manuscript at www.biomedcentral.com/submit
Biomed Central 